\title{
SURVIVING HYPOTHERMIA BY FERRITIN-MEDIATED IRON DETOXIFICATION
}

10 Tina Pekec ${ }^{1,2,6}$, Jaroslaw Lewandowski ${ }^{3,6}$, Alicja A. Komur ${ }^{3,6}$, Daria Sobanska ${ }^{3}$, Yanwu

11 Guo ${ }^{4}$, Karolina Świtońska-Kurkowska ${ }^{3}$, Marcin Frankowski ${ }^{5}$, Maciej Figiel ${ }^{3}$, and Rafal

12 Ciosk $^{3,4, *}$

$16{ }^{1}$ Friedrich Miescher Institute for Biomedical Research, Maulbeerstrasse 66, 4058 Basel,

17 Switzerland

182 University of Basel, Faculty of Natural Sciences, Klingelbergstrasse 70, 3026 Basel,

19 Switzerland

$20{ }^{3}$ Institute of Bioorganic Chemistry, Polish Academy of Sciences, Noskowskiego 12/14, 61-

21704 Poznań, Poland

$22{ }^{4}$ University of Oslo, Department of Biosciences, Blindernveien 31, Oslo, Norway

$23{ }^{5}$ Adam Mickiewicz University Poznan, Faculty of Chemistry, Uniwersytetu Poznańskiego 8,

24 61-614 Poznań, Poland

$25 \quad{ }^{6}$ These authors contributed equally

26

27 * Correspondence: rafal.ciosk@ibv.uio.no 


\section{SUMMARY}

29 How animals rewire cellular programs to survive cold is a fascinating problem with potential

30 biomedical implications, ranging from emergency medicine to space travel. Studying a

31 hibernation-like response in the free-living nematode Caenorhabditis elegans, we uncovered

32 a regulatory axis that enhances the natural resistance of nematodes to severe cold. This

33 axis involves conserved transcription factors, DAF-16/FoxO and PQM-1, which jointly

34 promote cold survival by upregulating FTN-1, a protein related to mammalian Fth1/ferritin.

35 Moreover, we show that inducing expression of Fth1 also promotes cold survival of

36 mammalian neurons, a cell type particularly sensitive to deterioration in hypothermia. Our

37 findings in both animals and cells suggest that FTN-1/Fth1 facilitates cold survival by

38 detoxifying ROS-generating iron species. We finally show that mimicking the effects of FTN-

39 1/Fth1 with drugs protects neurons from cold-induced degeneration, opening a potential

40 avenue to improved treatments of hypothermia.

41

42

\section{Keywords:}

44 Hypothermia, hibernation, cold, neuroprotection, FTN-1, Fth1, ferritin heavy chain,

45 ferroxidase, iron, $\mathrm{Fe}^{2+}$, ferrous iron, ROS, antioxidant, DAF-16, FoxO, PQM-1, ETS-4 


\section{INTRODUCTION}

48 Cold is a potentially lethal hazard. Nonetheless, hibernation is a widespread phenomenon, 49 used by animals to survive periods of low energy supply associated with cold ${ }^{1-4}$. Although 50 humans do not hibernate, some primates do so ${ }^{5}$, hinting that a hibernation-like state might,

51 one day, be induced also in humans, with fascinating medical repercussions ${ }^{6,7}$. Nowadays, 52 cooling is used widely in organ preservation for transplantation. Therapeutic hypothermia is 53 also applied, among others, during stroke or trauma, helping preserve functions of key 54 organs, like the brain or heart ${ }^{8,9}$. Cellular responses to cold are also of interest for longevity 55 research, as both poikilotherms (animals with fluctuating body temperature, like flies and 56 fish) and homeotherms (like mice) live longer at lower temperatures ${ }^{10,}{ }^{11}$. Therefore, 57 understanding the molecular underpinnings of cold resistance has the potential to transform 58 several areas of medicine.

The free-living nematode $C$. elegans populates temperate climates ${ }^{12}$, indicating that

60 these animals can survive spells of cold. In laboratories, C. elegans are typically cultivated 61 between $20-25^{\circ} \mathrm{C}$, and a moderate temperature drop slows down, but does not arrest, these 62 animals ${ }^{13,14}$. Deep cooling of $C$. elegans, i.e. to near-freezing temperatures, remains less 63 studied. Exposing nematodes to $2-4^{\circ} \mathrm{C}$, after transferring them directly from $20-25^{\circ} \mathrm{C}$ (which 64 we refer to as "cold shock"), results in the death of most animals within one day of 65 rewarming ${ }^{15-17}$. However, the lethal effects of cold shock can be prevented when animals 66 are first subjected to a transient "cold acclimatization/adaptation" at an intermittent 67 temperature of $10-15^{\circ} \mathrm{C}{ }^{15}, 17$. Such cold-adapted nematodes can survive near-freezing 68 temperatures for many days ${ }^{15,17-19}$. While in the cold, the nematodes stop aging, suggesting 69 that they enter a hibernation-like state ${ }^{17}$.

Among factors promoting $C$. elegans survival in near-freezing temperatures, we

71 identified a ribonuclease, REGE-1, homologous to the human Regnase-1/MCPIP1 ${ }^{17,20 .}$. In 72 addition to ensuring cold resistance, REGE-1 promotes the accumulation of body fat, which 
73 depends on the degradation of mRNA encoding a conserved transcription factor, ETS- $4{ }^{17}$.

74 Interestingly, previous studies showed that the loss of ETS-4 synergizes with the inhibition of

75 insulin signaling in extending lifespan ${ }^{21}$, and that the inhibition of insulin pathway 76 dramatically enhances cold survival ${ }^{15,19}$. Combined, these observations suggested that the 77 cold survival-promoting function of REGE-1 could be related to the inhibition of ETS-4/insulin 78 signaling axis. Here, we validate that hypothesis, dissect the underlying mechanism, and 79 demonstrate that its main objective is the detoxification of harmful iron species. We find that 80 a similar mechanism appears to protect from cold also mammalian cells. By mimicking its 81 effects with drugs, we highlight potential benefits of iron management for treating 82 hypothermia, for which no robust drug treatment currently exists.

83 


\section{RESULTS}

89

\section{Inhibition of ETS-4 improves $C$. elegans survival in the cold}

91 Our initial studies of $C$. elegans "hibernation" identified the RNase REGE-1 as a factor

92 promoting cold survival ${ }^{17}$. Studying REGE-1 in a different physiological context, the

93 regulation of body fat, we showed that a key target of REGE-1 encodes a conserved

94 transcription factor, ETS-4 ${ }^{17}$. Thus, we asked whether overexpression of ETS-4, taking

95 place in rege-1(-) mutants, is also responsible for the cold sensitivity of rege-1(-) mutants.

96 We tested that by incubating animals at $4^{\circ} \mathrm{C}$, henceforth simply the "cold" (for details on cold

97 survival assay see Fig. 1A). Indeed, we found that rege-1(-); ets-4(-) double mutants

98 survived cold much better than rege-1(-) single mutants (Fig. 1B). Unexpectedly, we

99 observed that the double mutants survived cold even better than wild type (Fig. 1B).

100 Intrigued, we additionally examined the ets-4(-) single mutants and found that they survived

101 cold as well as the double mutants (Fig. 1B). Thus, inhibiting ETS-4 is beneficial for cold

102 survival irrespective of REGE-1. This observation was somewhat surprising as, in wild type,

103 REGE-1 inhibits ETS-4 by degrading its mRNA. However, we observed that, in wild type,

104 both ETS-4 protein and ets-4 mRNA were more abundant in the cold (Fig. S1A-B). Thus, an

105 incomplete/inefficient degradation of ets-4 mRNA in the cold could explain the enhanced

106 cold survival of ets-4(-) mutants.

107 Because many hibernators burn fat to fuel survival in the cold, the ETS-4-mediated

108 fat loss ${ }^{17}$ and cold sensitivity (reported here), observed in rege-1(-) mutants, could be

109 connected. However, we found that inhibiting ETS-4 restores body fat of rege-1(-) mutants to

110 only wild-type levels ${ }^{17}$, and yet the rege-1(-); ets-4(-) double mutants are more resistant to

111 cold than wild type (Fig. 1B). We additionally examined the fat content of ets-4(-) single

112 mutants and found that it was indistinguishable from wild-type (Fig. 1C). Thus, ETS-4

113 appears to impact body fat and cold resistance via separate mechanisms. 
115 The enhanced cold survival requires both DAF-16 and PQM-1

116 ETS-4 was previously described to synergize with the insulin/IGF-1 signaling pathway in

117 limiting the nematode lifespan ${ }^{21}$. Moreover, the lifespan extension seen in ets-4(-) mutants,

118 as is the case with insulin pathway mutants, depends on the transcription factor DAF-

119 16/FOXO 21, 22. These and additional reports, that insulin pathway mutants display cold 120 resistance depending on DAF-16 ${ }^{15,19}$, prompted us to examine the genetic relationship 121 between ets-4(-) and insulin pathway mutants in the context of cold resistance. Firstly,

122 using a loss-of-function allele of the insulin-like receptor, daf-2(e1370) ${ }^{23}$, we confirmed that 123 inhibiting insulin signaling improves cold survival (Fig. S1C). Combining this daf-2 mutation 124 with ets-4(-), we observed that the double mutants survived cold slightly better than the daf-2 125 single mutant (Fig. S1C). However, the difference between daf-2(e1370); ets-4(-) and daf126 2(e1370) mutants was smaller than the difference between ets-4(-) mutant and wild type, 127 suggesting a partial overlap between mechanisms activated upon the inhibition of DAF-2 or 128 ETS-4.

129 The partial overlap suggests that ETS-4 may affect insulin signaling "downstream" 130 from the DAF-2 receptor. The main components of the C. elegans insulin pathway include 131 the phosphoinositide 3-kinase AGE-1/PI3K, which is why we also tested the genetic 132 relationship between age-1 and ets-4 mutations. Using the age-1(hx546) allele, carrying a 133 point mutation reducing the AGE-1 activity ${ }^{24}$, we confirmed that also the age-1(-) mutants 134 survive cold better than the wild type, and that their improved survival depends on the 135 transcription factor DAF-16/FOXO (Fig. S1D). Then, we examined the epistatic relationship 136 between age-1(hx546) and ets-4(-) mutants. While the age-1(hx546) single mutants survived 137 cold, expectedly, much better than wild type (Fig. 1D), we observed no additional benefit of 138 combining age-1(hx546) and ets-4(-) mutations (Figs. 1D and S1D). These observations 139 suggest that AGE-1 could act in the same pathway as ETS-4, or alternatively converge on 
140 the same downstream effector(s). Thus, we also examined whether the enhanced cold 141 survival of ets-4(-) mutants depends on DAF-16. Indeed, we found that removing DAF-16 142 completely suppressed the enhanced cold survival of ets-4(-) mutants (Fig. 1E). Reconciling 143 all observations, we hypothesize that, in wild type, signals generated upon DAF-2 or ETS-4 144 activation converge on AGE-1, thus inhibiting DAF-16 and limiting cold resistance. 145 Conversely, upon the inactivation of DAF-2 or ETS-4, DAF-16 activation results in improved 146 cold resistance.

147 Recently, another transcription factor, PQM-1, was shown to complement DAF-16 in 148 promoting the lifespan in DAF-2 deficient animals ${ }^{25}$. In the intestinal cells, PQM-1 and DAF14916 nuclear occupancy has been shown to be mutually exclusive, and they appear to regulate 150 largely separate sets of target genes ${ }^{25}$. Additionally, these transcription factors can play 151 opposing roles; for example, while the formation of an alternative "dauer" larval stage 152 (deployed to survive adverse environmental conditions) depends on DAF-16 ${ }^{22}, \mathrm{PQM}-1$ 153 facilitates the recovery from the dauer arrest ${ }^{25}$. Yet there is also some evidence supporting 154 synergistic roles of DAF-16 and PQM-1. For example, both the class I and II genes (see the 155 introduction) are down-regulated in pqm-1 mutants ${ }^{25}$, and DAF-16 and PQM-1 both 156 contribute to the lifespan extension of daf-2 or mitochondrial mutants ${ }^{25,26}$. Thus, at least in 157 certain circumstances, DAF-16 and PQM-1 may collaborate. Therefore, we tested whether 158 the loss of PQM-1 had a similar effect on the cold survival of ets-4(-) mutants as the loss of 159 DAF-16, and observed that, indeed, removing PQM-1 suppressed the enhanced cold 160 survival of ets-4(-) mutants (Fig. 1F). Importantly, in otherwise wild-type background, we 161 observed no apparent effects on cold survival in either pqm-1(-) or daf-16(-) single mutants, 162 nor in the pqm-1(-); daf-16(-) double mutants (Fig. S1E). Even the survival of daf-16(-); pqm163 1(-); ets-4(-) triple mutants was indistinguishable from wild-type nematodes (Fig. 1F). 164 Together, these observations argue for a specific, joint role for DAF-16 and PQM-1 in cold 165 survival, under conditions that favor their activation, such as upon ETS-4 inactivation. 
DAF-16 and PQM-1 are enriched in the gut nuclei in the cold

168 Presumably, DAF-16 and PQM-1 facilitate cold survival by inducing transcription of specific 169 genes. Under normal growth conditions, DAF-16 remains inactive in the cytoplasm. 170 However, when insulin signaling is inhibited, DAF-16 moves to the nucleus to activate target 171 genes. Based on the genetic analysis above, we suspected the nuclear accumulation of 172 DAF-16 in ets-4(-) mutants. To examine that, we attached (by CRISPR/Cas9 editing) a GFP173 FLAG tag to the C-terminal end of the endogenous daf-16 ORF (see Methods). Examining 174 the distribution of GFP-tagged DAF-16 (DAF-16::GFP), we observed little nuclear signal at $17520^{\circ} \mathrm{C}$, possibly with a minimal increase in the absence of ETS-4. After one or three days at $1764^{\circ} \mathrm{C}$, however, we observed a significant increase in the nuclear DAF-16::GFP (Fig. $2 \mathrm{~A}$ and $177 \mathrm{C}$ ); that increase appeared to be posttranscriptional, as daf-16 mRNA levels remained 178 constant between $20^{\circ} \mathrm{C}$ and $4^{\circ} \mathrm{C}$ (Fig. S2A). Although the nuclear DAF-16::GFP signal 179 appeared slightly stronger in ets-4(-) mutants at day one in the cold, that was no longer true 180 at day 3 (Fig. 2A and C). Thus, although the nuclear enrichment of DAF-16 is consistent with 181 its ability to potentiate cold resistance, that enrichment is, apparently, insufficient, as it only 182 enhances cold survival in ets-4(-) mutants but not wild type.

183 Thus, we performed the same analysis on PQM-1, fusing (by CRISPR/Cas9 editing) 184 an mCHERRY-MYC tag to the C-terminal end of the endogenous pqm-1 ORF (see 185 Methods). We detected little, if any, nuclear PQM-1::mCHERRY at $20^{\circ} \mathrm{C}$, in either wild-type 186 adult nematodes or ets-4(-) mutants (Fig. 2B-C), agreeing with the previously reported 187 expression patterns ${ }^{25,27-29}$. By contrast, after one day at $4^{\circ} \mathrm{C}$, we began detecting the 188 nuclear PQM-1::mCHERRY signal in wild-type nematodes, and a slightly stronger signal in 189 the nuclei of ets-4(-) mutants (Fig. 2B-C); this increase may be transcriptional, as pqm-1 190 mRNA levels were higher at $4^{\circ} \mathrm{C}$ than $20^{\circ} \mathrm{C}$ (Fig. S2B). After three days at $4^{\circ} \mathrm{C}$, the PQM191 1::mCHERRY nuclear signal increased even further (Fig. 2B-C) and, at this point, ets-4(-) 
192 mutants displayed significantly higher signal than wild type (Fig. 2B-C). Thus, in contrast to

193 the standard cultivation temperature, where DAF-16 and PQM-1 localize to the nucleus in a

194 mutually exclusive manner ${ }^{25}$, DAF-16 and PQM-1 coexist in the nucleus in the cold.

195 Intriguingly, DAF-16 and PQM-1 induce FTN-1 in ets-4 mutants but not wild type. Thus, one

196 possibility is that the additional accumulation of PQM-1 (observed in the absence of ETS-4)

197 is required to reach a threshold for DAF-16 activation. Among other possibilities, DAF-16

198 activation could involve additional factors normally inhibited by ETS-4.

Identification of a PQM-1 and DAF-16 coregulated gene promoting cold survival

201 The above observations are compatible with a scenario where, upon ETS-4 inactivation,

202 DAF-16 and PQM-1 coregulate transcription of cold survival-promoting gene(s). To test this

203 hypothesis, we undertook a functional genomic approach. First, we compared gene 204 expression (by RNAseq) between ets-4(-) and wild-type animals incubated at $4^{\circ} \mathrm{C}$. Then, by 205 comparing pqm-1(-); ets-4(-), or daf-16(-); ets-4(-) double mutants to the ets-4(-) single 206 mutant, we identified genes, whose expression in the ets-4(-) mutant depends on PQM-1 207 and/or DAF-16. To illustrate this, we prepared an integrative heat-map, using all $4^{\circ} \mathrm{C}$ 208 samples with replicates. Focusing on changes between the strains, we observed 3 distinct 209 clusters (Fig. S3A). Cluster 1 (red) includes genes upregulated in the cold in ets-4(-) mutants 210 (compared to wild type), which either do not change or go down, upon the additional 211 inhibition of daf-16 or pqm-1. Cluster 2 (green) includes genes upregulated across all 212 conditions. Finally, the smallest cluster 3 (blue), includes genes downregulated in ets-4(-) 213 mutants, which either do not change or go up, upon the additional inhibition of daf-16 or 214 pqm-1. With this analysis, we observed that many changes in gene expression upon the loss 215 of ETS-4, were reverted upon the additional loss of either DAF-16 or PQM-1, supporting a 216 functional relationship between DAF-16 and PQM-1. Taking advantage of the ENCODE 217 database, which reports genome-wide chromatin association of many transcription factors ${ }^{30}$, 
218 we examined the potential binding of DAF-16 and PQM-1 around the transcription start sites

219 (TSS) of genes in each cluster of the heat map. Even though the ENCODE data comes from

220 experiments performed at standard growth conditions, we decided to use it as an 221 approximation, and observed that genes, whose expression in ets-4(-) mutants depends on 222 DAF-16 or PQM-1 (i.e. genes in clusters 1 and 3), appear to be enriched for TSS-proximal 223 binding sites for both transcription factors (Fig. S3A). The same enrichment was not seen for 224 the cluster 2 genes, whose expression is apparently unrelated to DAF-16 or PQM-1 (Fig. 225 S3A). The possible connection between clusters 1 and 3, and the association with DAF-16 226 or PQM-1, was statistically significant for PQM-1 but not DAF-16 (Fig. S3A). Nevertheless, 227 by analyzing transcription factor binding motifs enriched within each gene cluster, we 228 observed a DAF-16-like motif enriched within cluster 1 genes (Fig. S3B), which made us 229 focus on those genes.

230 To identify candidate genes, whose DAF-16 and PQM-1 dependent activation 231 promotes cold survival, we first selected genes upregulated (in both biological replicates), at 232 least two-fold, in ets-4(-) mutants compared to wild type (after one day at $4^{\circ} \mathrm{C}$ ). Second, we 233 intersected these genes with those whose promoters associate with either DAF-1 or PQM-1, 234 according to the confident binding sites from Tepper et al. This analysis yielded seven genes 235 that were reproducibly upregulated in ets-4(-) mutants, and whose promoters may associate 236 with both PQM-1 and DAF-16 (Fig. 3A). If these genes were relevant for the enhanced cold 237 survival, their inhibition would be expected to impede cold survival of ets-4(-) mutants. 238 Testing this, we observed that RNAi-mediated depletion of one candidate, ftn-1 (encoding a 239 nematode ferritin), reproducibly compromised cold survival of ets-4(-) mutants (Fig. 3B; note 240 that the RNAi construct is predicted to target also ftn-2, which is highly similar to ftn-1, see 241 below). 
244 Iron is an essential but also a potentially harmful element, whose cellular levels are tightly 245 regulated by various molecular mechanisms, which are largely conserved between 246 nematodes and humans ${ }^{31,32}$. Among critical iron regulators are iron-binding proteins called

247 ferritins. In C. elegans, ferritin is encoded by two genes, ftn-1 and ftn-2. Under standard 248 growth conditions, ftn-2 mRNA is much more abundant than $\mathrm{ftn}-1^{33-35}$. While $\mathrm{ftn}-1$ was 249 previously shown to be upregulated upon DAF-2 inactivation, in a DAF-16 dependent 250 manner, ftn-2 is not considered a DAF-16 target ${ }^{36}$. Also, in agreement with their differential 251 regulation in innate immunity, the expression of $f t n-1$, but not $f t n-2$, was reported to depend 252 on PQM-1 ${ }^{28}$. Confirming our RNA profiling data by RT-qPCR, and consistent with the 253 regulation by both DAF-16 and PQM-1, we observed a DAF-16 and PQM-1 dependent 254 increase in the levels of ftn-1 mRNA in cold-treated ets-4(-) mutants (Fig. 3C-D). Because 255 the RNAi construct targets both $\mathrm{ftn}-1$ and $\mathrm{ftn}-2$, we also examined FTN-1 function using an 256 existing loss-of-function allele, ftn-1(ok3625) ${ }^{37,38}$. Since DAF-16 and PQM-1 are important 257 for cold survival in ets-4(-), but not wild-type animals, their relevant target may be expected 258 to display a similar behavior. Indeed, the cold survival of $f t n-1(-)$ single mutants was 259 indistinguishable from wild type (Fig. 4A), but the ftn-1 inactivation completely abolished the 260 enhanced cold survival of ets-4(-) mutants (Fig. 4A). Importantly, FTN-1 is expressed in the 261 intestine ${ }^{34}$, i.e. the tissue where ETS-4, DAF-16 and PQM-1 are all expressed.

262 If the cold survival-enhancing role of FTN-1 is related, as expected, to its function in 263 iron regulation, excess iron may be expected to impede cold survival. We tested that by 264 supplementing culture plates with ferric ammonium citrate (FAC). Although we do not know 265 how much extra iron is being absorbed by FAC-treated animals, we observed a dose266 dependent impediment of cold survival (Fig. S4A). Importantly, although higher FAC levels 267 reduced the survival of ets-4(-) mutants, their survival was still greater than of the 268 corresponding (i.e. FAC-treated) wild type (Fig. S4B). The better survival of FAC-treated ets269 4(-) mutants still depended on FTN-1, as the ftn-1(-); ets-4(-) double mutants responded to 
270 excess iron like the corresponding wild type (Fig. S4B). Combined, our data suggest that

271 FTN-1, when expressed in cold-treated ets-4(-) animals, facilitates survival, and its beneficial

272 effect involves some form of iron regulation.

\section{FTN-1 promotes cold survival through its ferroxidase activity}

275 Mammalian ferritin consists of multiple heavy and light subunits (FTH and FTL) that form 276 nanocages storing thousands of iron atoms ${ }^{39}$. The C. elegans ferritins, FTN-1 and -2 , are

277 more similar to $\mathrm{FTH}^{33}$. To test iron sequestration by $\mathrm{FTN}-1$, we used size exclusion chromatography-inductively coupled plasma-mass spectrometry (SEC-ICP-MS; ${ }^{38}$ ). We found that, in cold-treated animals, the levels of total iron were independent of FTN-1 (Figs. 4B, S4C, E) but, as reported at standard temperature ${ }^{38}$, depended on FTN-2 (Figs. 4C, S4D, E). Thus, FTN-1 appears to contribute little, if at all, to the pool of stored iron.

Iron is present in cells in both oxidized $\mathrm{Fe}^{3+} /$ ferric(III) and reduced $\mathrm{Fe}^{2+} /$ ferrous(II)

283 forms. Excess of $\mathrm{Fe}^{2+}$ is potentially harmful, because, in the so-called Fenton reaction, it 284 catalyzes the formation of reactive oxygen species, ROS ${ }^{40,41}$. Notably, both FTN-1 and -2 contain predicted ferroxidase active sites, which in homologous proteins mediate the $\mathrm{Fe}^{2+}$-to$\mathrm{Fe}^{3+}$ conversion (Figs. 4D and S4F). Accordingly, the ftn-2; ftn-1 double mutants display an elevated ratio of $\mathrm{Fe} 2+/ \mathrm{Fe} 3+$ during aging ${ }^{38}$. Although the individual impact of $\mathrm{FTN}-1$ on the $\mathrm{Fe} 2+/ \mathrm{Fe} 3+$ balance was not examined, the overexpression of $\mathrm{ftn}-1$ was reported to have antioxidant effects ${ }^{42}$. To test whether the ferroxidase activity of FTN-1 promotes cold survival, we modified the endogenous ftn-1 locus (by CRISPR/Cas9 editing), so that it produces a ferroxidase-dead FTN-1. Crucially, inactivation of the FTN-1 ferroxidase activity completely abolished the enhanced cold survival of ets-4(-) animals (Fig. 4E). Thus, FTN-1 appears to facilitate cold survival through iron detoxification, rather than sequestration. 
296 Thus far, we have shown that FTN-1, when expressed in the absence of ETS-4, gives 297 worms advantage in surviving cold. To test whether FTN-1 may do that in otherwise wild298 type background, we created (using Mos1-mediated Single Copy Insertion, MosSCl; ${ }^{43}$ ) strains overexpressing $f t n-1$ from two different, robust promoters, $d p y-30$ and vit-5.

300 Importantly, we found that both strains survived cold much better than wild type (Fig. 5A; for 301 the levels of ftn-1 mRNA overproduced from the vit-5 promoter see Fig. S5A). By SEC-ICP-

302 MS, we observed no changes in the levels of ferritin-associated iron in the overexpressing 303 strains (Figs. 5B and S5B, C), consistent with FTN-1 functioning in iron detoxification and 304 not sequestration.

The ferroxidase activity of FTN-1 is expected to lower the levels of ROS-generating Fe(II), implying that cold-treated nematodes experience increased levels of ROS. We found 307 that reagents typically used for ROS detection (like CM- $\mathrm{H}_{2}$ DCFDA) are toxic to cold-treated 308 animals. Thus, we sought a factor whose induction could be used as a proxy for ROS 309 detection. Specific enzymes, called superoxide dismutases (SODs), function at the front line 310 of cellular defense against ROS ${ }^{44}$. There are five SODs in C. elegans and, examining their 311 expression in cold-treated animals, we noticed a consistent increase in the sod-5 mRNA. To 312 understand the dynamics of sod-5 activation, we examined the expression of GFP-tagged 313 SOD $-5^{45}$; the fusion protein is expressed mainly in neurons. Following SOD-5::GFP signal in 314 live animals, we observed a strong, but transient increase of SOD-5::GFP during rewarming 315 (Fig. S5D; note the elevated signal around $2 \mathrm{~h}$ into rewarming). Focusing thus on this time 316 point, we tested whether the overexpression of $f t n-1$ impacts sod-5 activation. Indeed, we 317 observed that the levels of sod-5 mRNA were significantly lower in the ftn-1 overexpressing 318 strain than wild type (Fig. 5C). All observations combined, a picture emerges where FTN-1, 319 through its iron(II)-detoxifying activity, protects animals from the cold by reducing the levels 320 of $\mathrm{Fe}(\mathrm{II})$-catalyzed ROS. According to this model, animals subjected to cold experience an 321 increase in $\mathrm{Fe}(\mathrm{II})$ iron. Detection of specific iron forms is not trivial, and our attempts to 
322 detect specifically $\mathrm{Fe}(\mathrm{II})$ in $\mathrm{C}$. elegans were unsuccessful. Thus, assuming some level of

323 conservation in cellular responses to cold, we decided to investigate that in mammalian

324 cells, where $\mathrm{Fe}(\mathrm{II})$ detection is more robust and potential findings more appllicable to human

325 hypothermia.

327 Iron management plays a key role in neuronal resistance to cold

328 Since the main clinical benefit of deep cooling is the preservation of neuronal functions, we 329 decided to examine $\mathrm{Fe}(\mathrm{II})$ in neurons, where our observations may be of clinical relevance.

330 For convenience, we chose to study murine neurons. To generate them, we differentiated 331 primary neuronal stem cells, collected from early mouse embryos, into noradrenergic-like 332 neurons (henceforth "neurons"), which affect numerous physiological functions, generally 333 preparing the body for action. To examine their cold resistance, neurons (cultivated at the 334 physiological temperature of $37^{\circ} \mathrm{C}$ ) were shifted to $10^{\circ} \mathrm{C}$ for 4 hours and then returned to $33537^{\circ} \mathrm{C}$. Their viability was examined after rewarming for 24 hours (see Methods for details).

336 First, we observed that cooling induced cell death in a large fraction of neurons (Fig. 6A). 337 Interestingly, neuronal death was associated with rewarming (Fig. S6A), which is somewhat 338 reminiscent of reperfusion injury, arguing that not the cold per se, but rather the burden 339 associated with restoring cellular functions during rewarming, is the critical challenge facing 340 cold-treated neurons.

341 A recent study compared cold survival of neurons derived from either hibernating or 342 non-hibernating mammals, and reported that "hibernating" neurons survive cold much better 343 than "non-hibernating" ones ${ }^{46}$. Thus, hibernating neurons appear to possess intrinsic 344 mechanisms enhancing cold resistance. Remarkably, treating non-hibernating neurons with 345 certain drugs was shown to compensate, at least partly, for their lower cold resistance.

346 Although house mice, upon starvation, are capable of daily torpor ${ }^{47}$, they can be considered 347 as non-hibernators in a classical sense. Correspondingly, we observed that treating murine 
neurons with either BAM15 or PI (drugs previously used by Ou et al.) increased their cold survival (Fig. 6A). Assuming that, like for nematodes, iron management is crucial for the survival of cold-treated neurons, we treated neurons with the iron-chelating drug deferoxamine, DFO (expected to lower cellular levels of free iron). Indeed, DFO treatment protected neurons from cold-related death to the same extent as BAM15, PI, or drug combinations (Fig. 6A).

In contrast to hibernating neurons, the non-hibernating neurons display a striking deterioration of neuronal processes/neurites, which is counteracted by BAM15 and/or PI treatment ${ }^{46}$. By staining neurons against NEFH (neurofilament protein heavy polypeptide; a neuron-specific component of intermediate filaments), we observed that also DFO had a strong stabilizing effect on cold-treated neurites (Fig. 6B-C). This protection appeared to be long-lasting, as the neurites were still evident at $24 \mathrm{~h}$ into rewarming (Fig. S6B-C).

\section{Overproduction of FTH1 improves cold survival of mammalian neurons}

362 By lowering the pool of free iron, DFO could, indirectly, reduce the levels of $\mathrm{Fe}(\mathrm{II})$. 363 Nonetheless, to monitor ferrous iron directly, we employed a fluorescent probe, FeRhoNox364 1, which specifically detects $\mathrm{Fe}(\mathrm{II})$. Strikingly, we observed a strong, though transient, 365 increase of $\mathrm{Fe}(\mathrm{II})$ during rewarming (Fig. S7A-B), which was prevented by DFO treatment 366 (Fig. 7A). Since ferrous iron catalyzes the formation of reactive oxygen species (ROS), we 367 also measured ROS levels, using CellROX-green. We observed a strong increase of ROS 368 during rewarming, at the time coinciding with the Fe(II) peak. Importantly, that increase was counteracted by DFO treatment, as expected (Fig. 7B).

If, as expected, decreasing ROS is important for the recovery from cold, treating 371 neurons with antioxidants should provide similar benefit as DFO. To test that, we selected

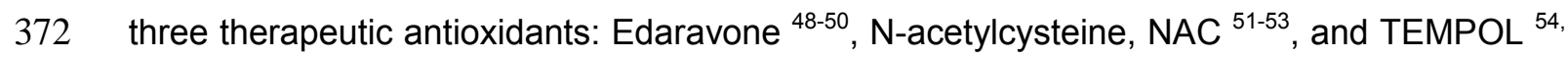

37355 . Indeed, treating neurons with these drugs strongly enhanced cold survival (Fig. 7C). 
374 Finally, we decided to test whether, similar to ftn-1 overexpression in nematodes,

375 overexpression of its mammalian counterpart, Fth1, improves cold survival of neurons.

376 Indeed, we found that Fth1-overexpressing neurons survived cold significantly better than

377 mock-transfected neurons (Fig. 7D).

378 Summarizing, cultured neurons appear to respond to hypothermia in a manner 379 remarkably reminiscent of nematodes. Although $\mathrm{Fe}(\mathrm{II})$ was only imaged in neurons, both 380 nematodes and neurons display a transient increase in ROS during rewarming. Moreover, 381 induction of FTN-1/FTH1 enhances cold survival in both models. Presumably, this reflects

382 the capacity of both orthologous proteins for iron detoxification and, consequently, for 383 reducing ROS. Importantly, targeting the free iron-Fe(II)-ROS axis with drugs enhances 384 neuronal cold resistance, suggesting that these and related drugs might prove beneficial in 385 treating hypothermia. 


\section{DISCUSSION}

389 Using a simple, genetically tractable animal model for cold preservation, we uncovered a 390 conserved ability of ferritin to promote cold survival. In this context, FTN-1/FTH1 (ferritin 391 heavy chain) appears to function as an endogenous antioxidant, which counteracts iron392 mediated cytotoxicity arising during the recovery from cold. Intriguingly, the elevated 393 expression of $F T H 1$ has been recognized as a distinctive feature of cold adaptation in 394 hibernating primates, during both daily torpor and seasonal hibernation ${ }^{56,57}$. Thus, a mechanism that we turned on artificially in nematodes, through genetic manipulation, may

396 be used actually by some hibernators as a cell-intrinsic mechanism boosting cold resistance.

397 What causes in those animals FTH1 upregulation remains unknown. However, FoxO3a 398 (related to DAF-16 described here) is upregulated in hibernating squirrels ${ }^{58}$, arguing for a conserved function of FoxO transcription factors in cold resistance, which might involve the induction of ferritin.

How exactly, and why, cold triggers the accumulation of toxic iron remains to be 402 fully understood. In mammalian epithelial cells, a sizeable fraction of cold-induced free iron 403 was proposed to originate from the microsomal cytochrome P-450 enzymes, which require 404 iron-containing heme as a cofactor ${ }^{59}$. It was also suggested that the cytosolic free iron 405 causes mitochondrial permeabilization, resulting in apoptosis ${ }^{60}$. However, as also shown 406 here, treating neurons with BAM15, a mitochondrial uncoupler, suppresses both ROS and 407 cold-induced death ${ }^{46}$. Assuming that the same treatment reduces iron(II) levels, it may 408 instead suggest the mitochondrial origin of iron toxicity, which agrees with the general view 409 that most cellular ROS is of mitochondrial origin ${ }^{61}$. Irrespective of its sources, once 410 accumulating, iron(II) can catalyze the formation of ROS, damaging diverse cellular 411 components (like lipids, proteins and nucleic acids), and causing a number of acute and 412 chronic degenerative conditions ${ }^{62,63}$. 
413 Crucially, the antioxidant defense is a hallmark of hibernation, being particularly

414 critical during the entry to and exit from hibernation, when oxygen-sensitive tissues, like the 415 brain, are particularly vulnerable to ischemia/reperfusion injury ${ }^{4}$. Thus, the challenges 416 associated with cooling appear to be, to some extent, similar to those facing the brain in 417 other pathologies. Indeed, the antioxidants employed here have been used to improve 418 outcomes of acute ischemic stroke (Edaravone), hypoxic-ischemic encephalopathy (NAC), 419 and iron-induced cerebral ischemic injury (TEMPOL). Therefore, activating mechanisms 420 protecting cells from cold, or mimicking their effects with drugs, could benefit not only 421 hypothermia patients, but be potentially useful in treating other pathologies, like stroke or 422 neurodegenerative disorders. 


\section{ACKNOWLEDGMENTS}

425 We are grateful to Susan Gasser and the Gasser lab for supporting TP in later stages of her

$426 \mathrm{PhD}$. We thank S. Smallwood and S. Thiry for assistance with mRNA sequencing, L.

427 Gelman and S. Bourke for imaging support, and W. Wendlandt-Stanek for FTN-1 modeling.

428 We thank Collin Ewald, Jacek Kolanowski, Gawain McColl, and Göran Nilsson for 429 discussions and comments on the manuscript. The project POIR.04.04.00-00-203A/16 was 430 carried out within the Team program of the Foundation for Polish Science, co-financed by 431 the European Union under the European Regional Development Fund. RC was also 432 supported by the EMBO Installation Grant No. 3615, the Polish National Science Centre 433 grant 2019/34/A/NZ3/00223, and the Research Council of Norway grant FRIMEDBIO434 286499. KS-K and MFi were supported by the National Science Centre grant $4352018 / 31 / B / N Z 3 / 03621$. Some of the strains were provided by the Caenorhabditis Genetics 436 Center (CGC) funded by the NIH. The Nestin antibody was obtained from the 437 Developmental Studies Hybridoma Bank, created by the NICHD of the NIH, and maintained 438 at the University of lowa.

\section{AUTHOR CONTRIBUTIONS}

441 TP performed and analyzed most nematode experiments in Figs. 1-3. JL and KŚ-K 442 performed and analyzed experiments in Figs. 6-7, under the guidance of MFi, who also 443 provided the neuronal stem cell-sphere model. AK and DS performed and analyzed 444 nematode experiments shown in Figs. 4-5; MFr oversaw the ICP-MS experiments. YG 445 analyzed the genomic data, and performed some nematode experiments. RC conceived and 446 supervised the project. RC with other authors wrote the manuscript.

\section{DECLARATION OF INTERESTS}

449 The authors declare no competing interests. 
Pekec et al., Figure 1

A

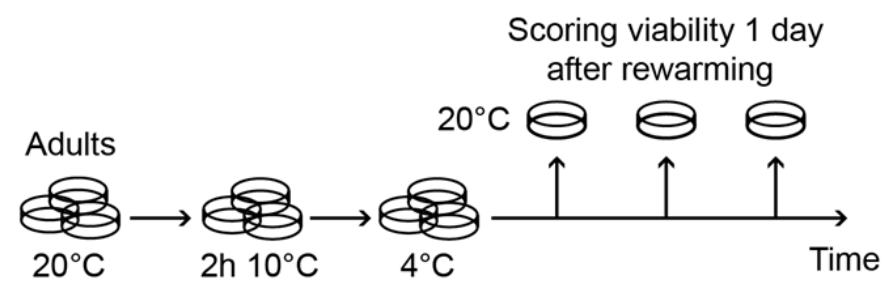

B
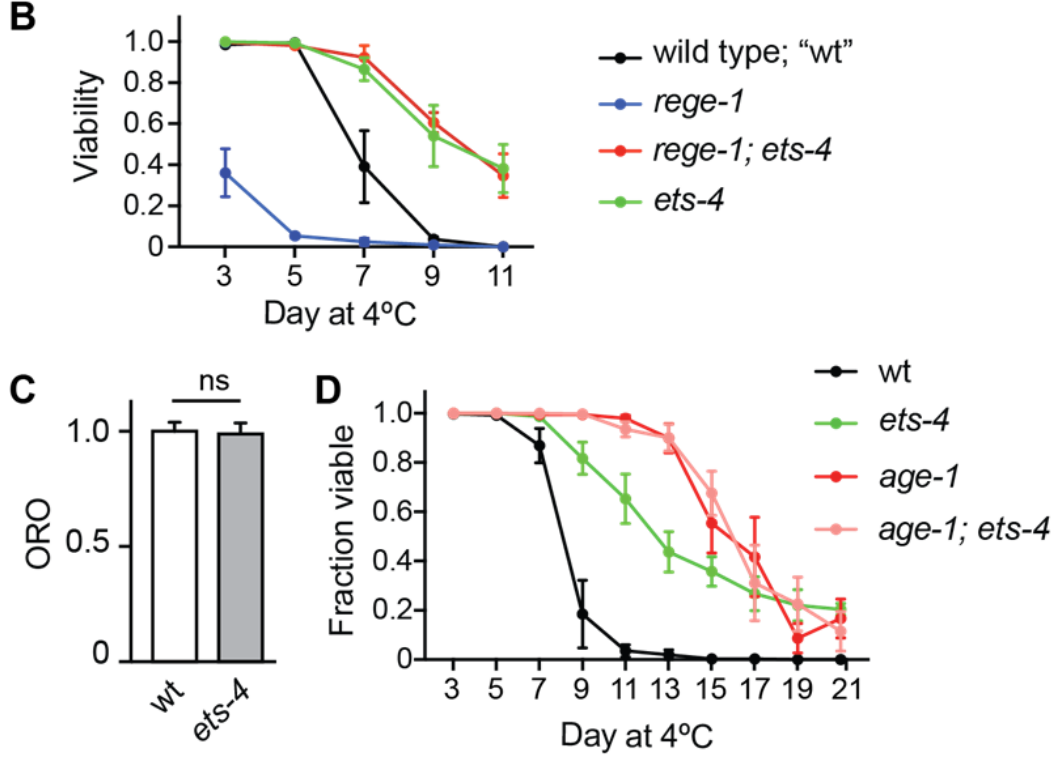

E

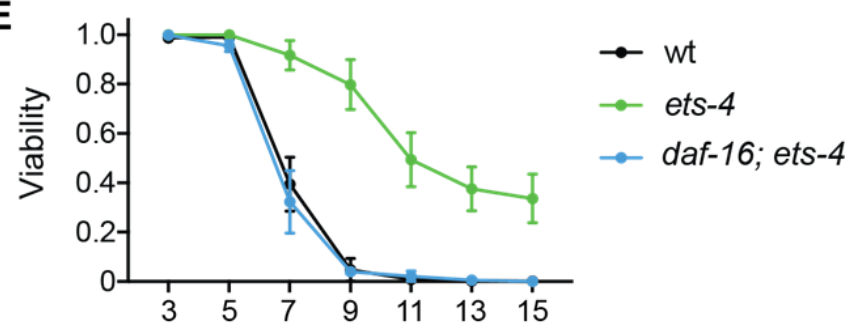

Day at $4^{\circ} \mathrm{C}$

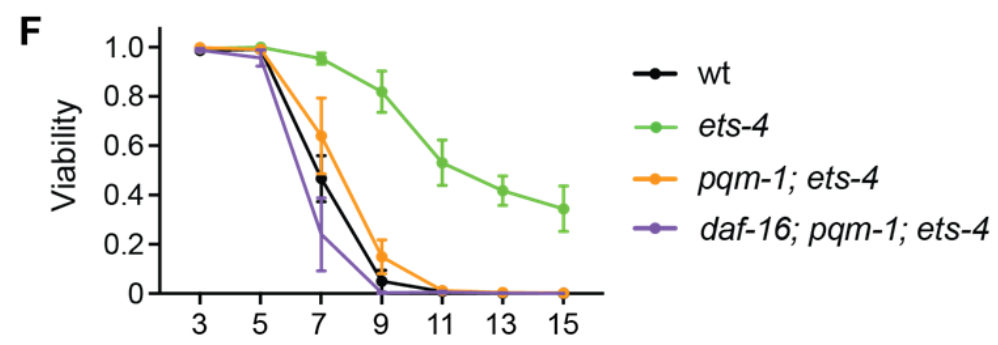

Day at $4^{\circ} \mathrm{C}$ 
453 Figure 1. Enhanced cold survival of ets-4 (-) mutants depends on DAF-16 and PQM-1

454 A. Graphical view of a typical cold-survival experiment, described in detail in the Methods.

455 Shortly, 1 day-old adults, pre-grown at $20^{\circ} \mathrm{C}$ and distributed between multiple plates, were

456 cold-adapted (for 2 hours at $10^{\circ} \mathrm{C}$ ) and then shifted to $4^{\circ} \mathrm{C}$. Every few days, a plate was

457 transferred to $20^{\circ} \mathrm{C}$ and, after 1 day of recovery at $20^{\circ} \mathrm{C}$, the animals were scored for 458 viability.

459 B. Cold survival of animals of the indicated genotypes. Mutant alleles throughout the paper 460 are indicated in parentheses and, unless specified otherwise, are loss-of-function alleles. 461 Note that ets-4(rrr16) mutants, like rege-1(rrr13); ets-4(rrr16) double mutants, survived cold 462 better than wild type (wt). The experiment was performed three times $(n=3) ; 200-350$ 463 animals were scored per time point. Error bars represent standard error of the mean (SEM).

464 C. Quantification of body fat, stained with the lipophilic dye oil red O (ORO), in animals of the 465 indicated genotypes. The levels of body fat were similar between wt and ets-4(rrr16) 466 mutants. $n=3 ; 10-15$ animals were scored per replicate. Error bars represent SEM. Unpaired 467 two-tailed t-test was used to calculate the $p$ value, "ns" = not significant.

468 D. Cold survival of animals of the indicated genotypes (D-F; error bars represent SEM). The 469 age-1(hx546) mutants displayed greatly enhanced cold resistance, and combining age$4701(h \times 546)$ and ets-4(rrr16) mutations did not provide animals with additional resistance. $\mathrm{n}=4$; $471 \quad 350-500$ animals were scored per time point.

472 E. Combining daf-16(mu86) and ets-4(rrr16) mutations abolished the enhanced cold survival 473 of ets-4(-) mutants, reverting it to wild-type values. $n=4 ; 350-500$ animals were scored per 474 time point.

475 F. Combining pqm-1(ok485) and ets-4(rrr16) mutations abolished the enhanced cold survival 476 of ets-4(-) mutants, reverting it to wild-type values. Also note that the triple daf-16(mu86); 477 pqm-1(ok485); ets-4(rrr16) mutants survived cold essentially like wild type, indicating that 
bioRxiv preprint doi: https://doi.org/10.1101/2021.01.30.428937; this version posted February 1, 2021. The copyright holder for this preprint (which was not certified by peer review) is the author/funder. All rights reserved. No reuse allowed without permission.

478 DAF-16 and PQM-1 promote cold survival in ets-4(-), but not wild-type animals. $\mathrm{n}=4$; 450 479650 animals were scored per time point. 
Pekec et al. Figure 2

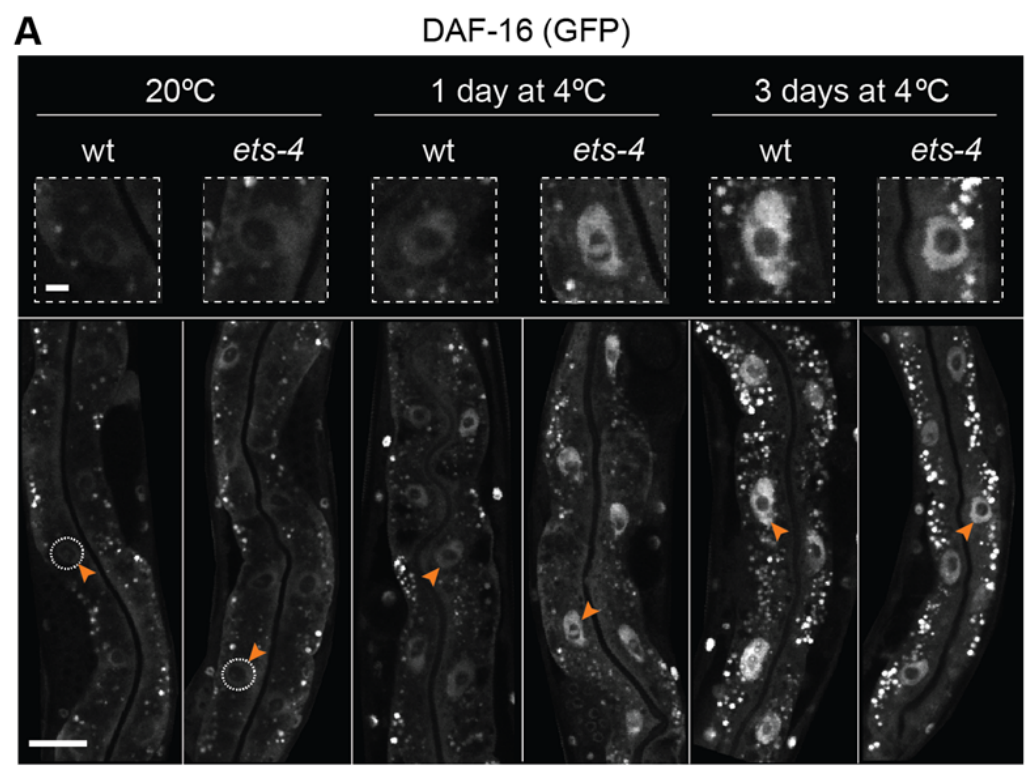

B

PQM-1 (mCHERRY)
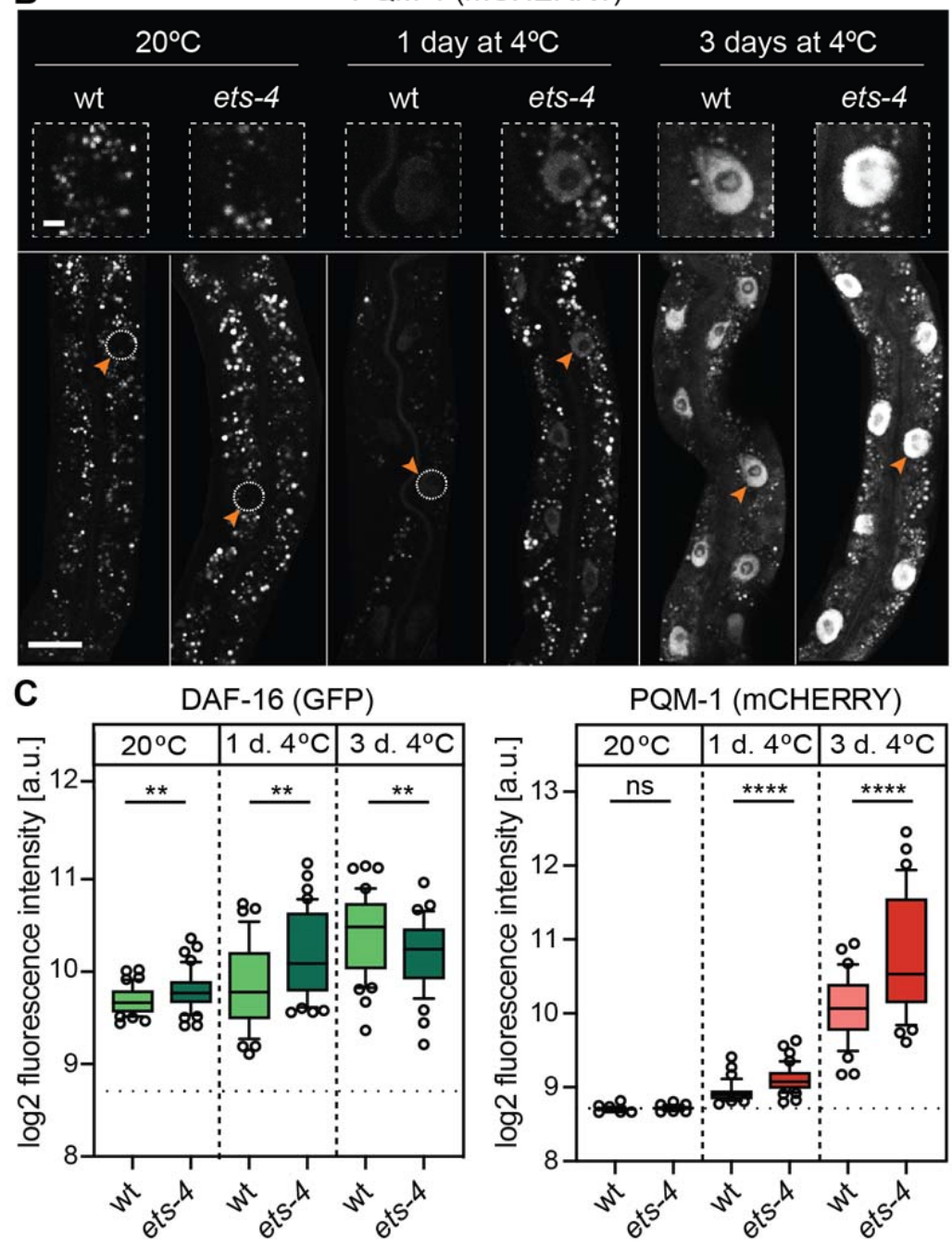
481 Figure 2. DAF-16 and PQM-1 are enriched in the nuclei in the cold

482 A. Micrographs showing representative confocal images of GFP fluorescence, reflecting the 483 endogenously tagged DAF-16, allele daf-16(syb707), from wt or ets-4(rrr16) mutants. The 484 animals were sampled at the indicated times and temperatures, according to $1 \mathrm{~A}$. The 485 corresponding quantifications are in C. Arrowheads point to representative gut nuclei 486 (demarcated with dashed circles when displaying little or no fluorescence), which are 487 enlarged in the insets above. Size bars, here and in B: $5 \mu \mathrm{m}$ (small magnification) and 25 $488 \mu \mathrm{m}$ (large magnification).

489 B. Representative confocal images of mCherry fluorescence, reflecting the endogenously 490 tagged PQM-1, allele pqm-1(syb432), from wt or ets-4(rrr16) mutants. The animals were 491 sampled as above. The corresponding quantifications are in C. Arrowheads point to 492 representative gut nuclei, enlarged in the insets above.

493 C. Quantifications of the nuclear fluorescence, corresponding to A (left) and B (right). Each 494 data point represents $\log _{2}$-transformed mean nuclear intensity per animal. Dotted line 495 represents the average background within each experiment. Left: $n=3 ; 10$ to 15 animals 496 were scored per replicate. Error bars represent $10^{\text {th }}$ to $90^{\text {th }}$ percentile. Unpaired two-tailed t497 test was used to calculate the $p$ value. ${ }^{* *}$ indicates $p<0.01$. Right: $n=3 ; 10$ to 15 animals 498 were scored per replicate. "ns" = not significant; ${ }^{* * * *}$ indicates $p<0.0001$. 
Pekec et al. Figure 3
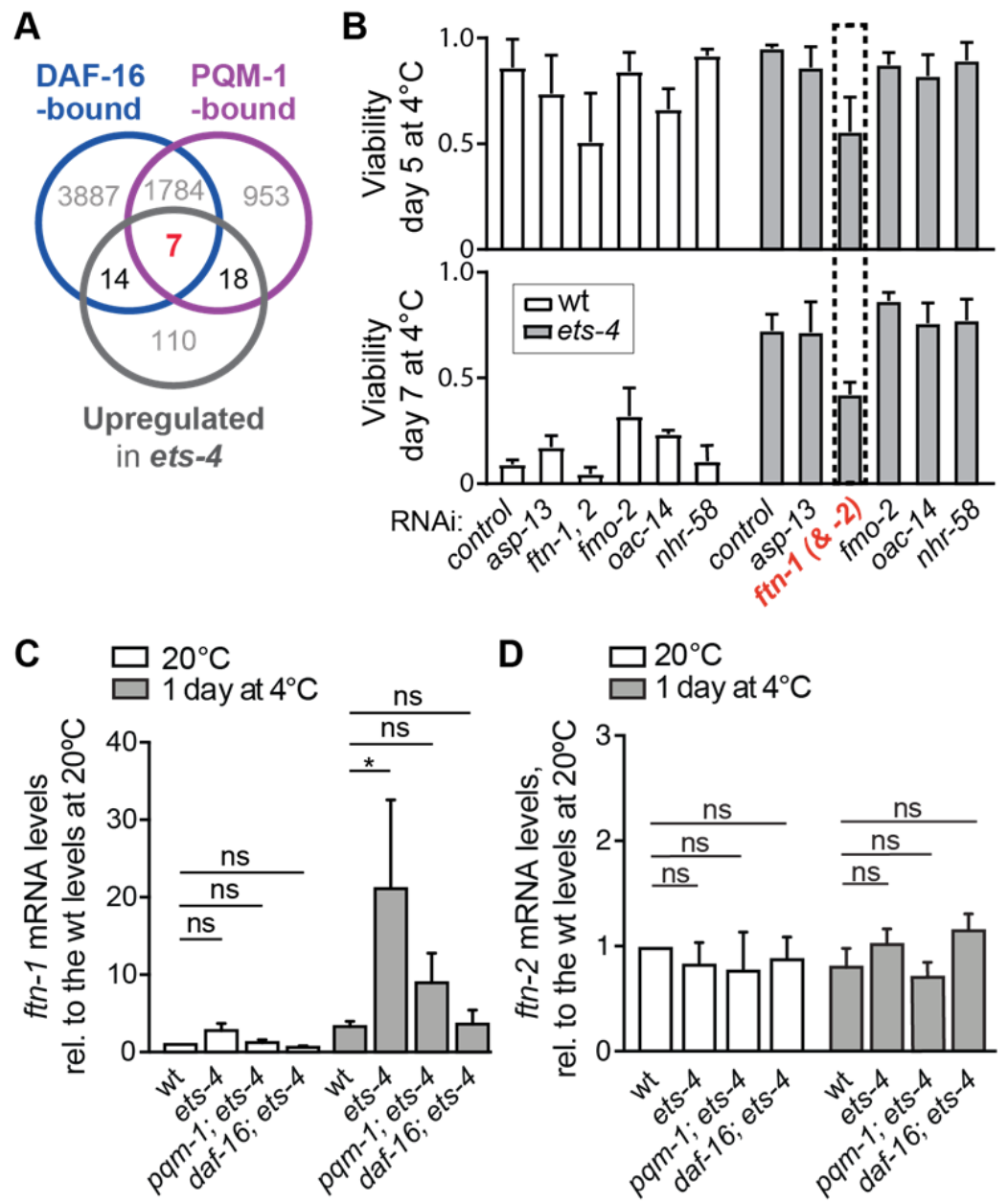

\section{Figure 3. DAF-16 and PQM-1 coregulate expression of ftn-1/ferritin}

502 A. Diagram comparing relations between three sets of genes: Grey circle: genes upregulated more than 2-fold in ets-4(rrr16) mutants compared to wt (at day 1 at $4^{\circ} \mathrm{C}$ and in

504 two replicates, see Fig. S3A). Blue circle: genes whose promoters are bound by DAF-16

505 (according to Tepper et al.). Magenta: genes whose promoters are bound by PQM-1

506 (according to Tepper et al.). Note that seven genes (asp-13, cpt-4, fmo-2, ftn-1, nhr-58, oac-

50714 , and pals-37), whose promoters are bound (at normal temperature) by both DAF-16 and 508 PQM-1, were reproductively upregulated in the absence of ETS-4. 
509 B. The candidates from A were RNAi-depleted, from either wt or ets-4(rrr16) animals, and 510 those animals were tested for cold resistance (according to $1 \mathrm{~A}$ ) at the indicated times. Note 511 that depleting $f t n-1$ (and ftn-2, as RNAi is predicted to target both homologs) significantly 512 reduced cold survival of ets-4(rrr16) animals (stippled box). Error bars represent SEM. $\mathrm{n}=3$;

$513 \quad 200-350$ animals were scored per time point.

514 C. Shown are ftn-1 mRNA levels, measured by RT-qPCR, in animals of the indicated 515 genotypes. Strains used: wt, ets-4(rrr16), pqm-1(ok485); ets-4(rrr16), and daf-16(mu86); ets5164 ( $r r r 16)$. The animals were sampled at $20^{\circ} \mathrm{C}$, before cold adaptation, and after one day at $5174^{\circ} \mathrm{C}$, according to $1 \mathrm{~A}$. The mRNA levels were normalized to the levels of act-1 (actin) mRNA. 518 At each temperature, the values were then normalized to those from the wild type at $20^{\circ} \mathrm{C}$. $519 \mathrm{n}=5$; error bars represent SEM. $\mathrm{P}$ values were calculated using 2-way ANOVA for multiple 520 comparisons. "ns" = not significant; ${ }^{*}$ indicates $p>0.05$.

521 D. The animals were collected as in C. The levels of ftn-2 mRNA, measured by RT-qPCR, 522 were normalized to act-1 mRNA, and are shown relative to the ftn-2 mRNA level in wt at $52320^{\circ} \mathrm{C}$. Wt and ets- $4(-)$ animals were collected at $20^{\circ} \mathrm{C}$ and after one day at $4^{\circ} \mathrm{C}(\mathrm{n}=3) . \mathrm{P}$ 524 values were calculated using 2- way ANOVA. Error bars represent SEM. "ns" = not 525 significant. 
Pekec et al. Figure 4
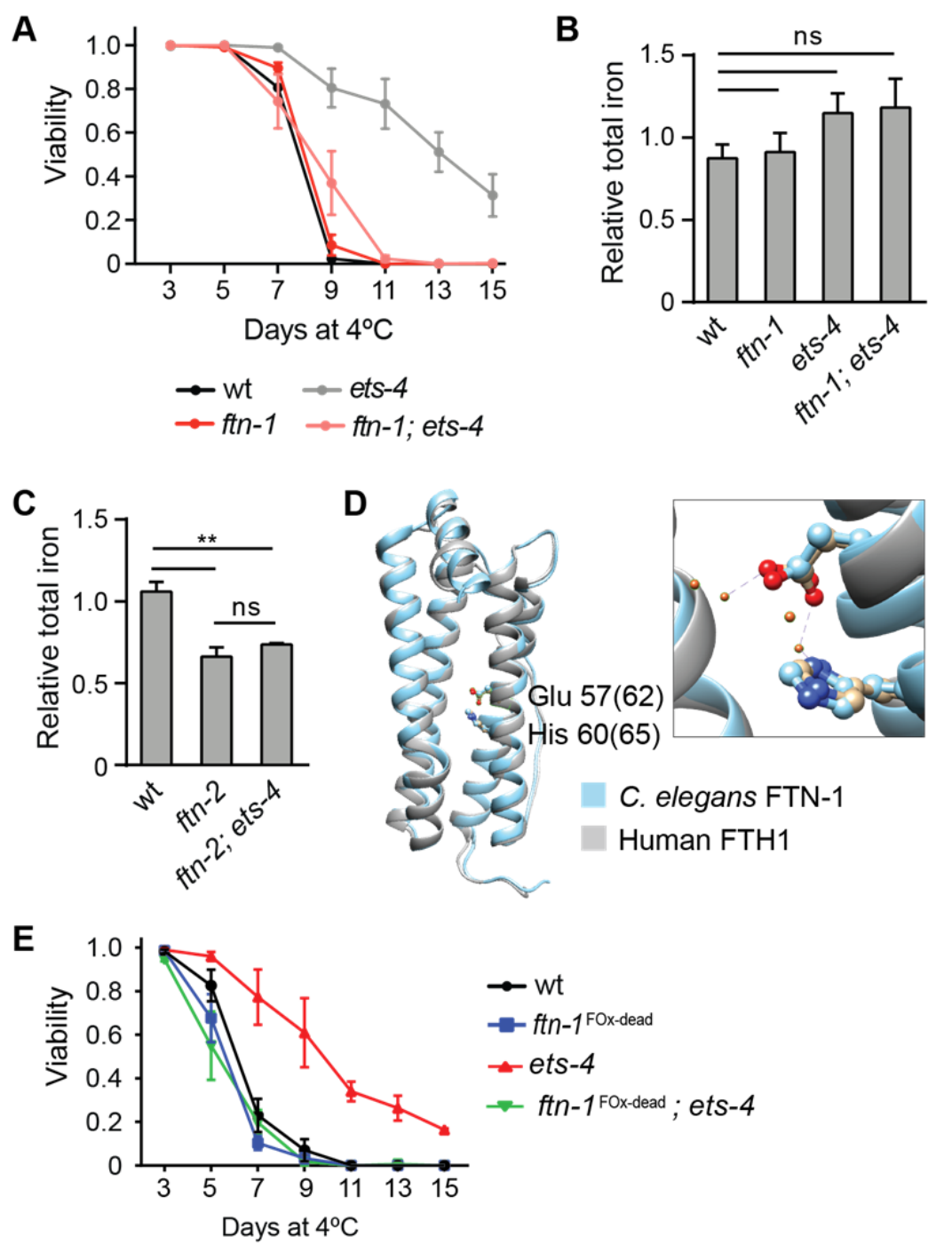

Days at $4^{\circ} \mathrm{C}$

\section{Figure 4. FTN-1 promotes cold survival via its ferroxidase activity}

529 A. Viability of animals, of the indicated genotypes, subjected to cold as explained in $1 \mathrm{~A}$.

530 Error bars represent SEM. $n=3 ; 250-400$ animals were scored per time point. Note that

531 combining the $f t n-1$ (ok3625) mutation with ets-4(rrr16) reverted the enhanced cold survival

532 of ets-4(-) mutants to wild-type values, similar to the double daf-16(-); ets-4(-) or pqm-1(-);

533 ets-4(-) mutants in 1E-F. Also, like daf-16(-) and pqm-1(-) single mutants in S1E, ftn-

5341 (ok3625) single mutants survived cold as well as wt. 
535 B. Total iron levels in C. elegans extracts, measured by ICP-MS. Wild type, ets-4(rrr16), ftn536 1(ok3625), and ftn-1(ok3625); ets-4(rrr16), 1 day-old adults were subjected to cold for 3 537 days. The values were normalized to those of the wild type. Unpaired two-tailed t-test was 538 used to calculate the $p$ value, "ns" = not significant. Error bars represent SEM, $n=3$. Note 539 that inactivating FTN-1 had no impact on the total iron levels.

540 C. Comparing total iron levels, measured by ICP-MS, in C. elegans extracts derived from 541 wild-type, ftn-2(ok404), and ftn-2(ok404); ets-4(rrr16), 1 day-old adults, subjected to cold for 5423 days. The values were normalized to those of the wild type. Unpaired two-tailed t-test was 543 used to calculate the $p$ value, "ns" $=$ not significant, ${ }^{* *} p$ value $<0.01$. Error bars represent 544 SEM, $n=3$. Note that inactivating FTN-2 decreased the levels of total iron, also in ets-4(-) 545 animals.

546 D. Structural alignment of $H$. sapiens ferritin heavy chain 1 (FTH1 - colored in grey, PDB 547 code 4 OYN) and C. elegans FTN-1 (colored in light blue), using the Phyre2 tool ${ }^{64}$. Amino 548 acids critical for the ferroxidase activity are shown as balls and sticks. The colors indicate: 549 carbon atoms in FTH1 (light brown) or FTN-1 (light blue), oxygen atom of glutamic acid 550 (red), and nitrogen atom of histidine (dark blue). The magnification shows the ferroxidase 551 active site, with the iron atoms shown as dark orange balls and coordination bonds as dotted 552 lines.

553 E. Cold survival of animals of the indicated genotypes subjected to cold, as in $1 \mathrm{~A}$. "ftn-1 ${ }^{\text {FOx- }}$ 554 dead" indicates the ftn-1(syb2550) allele, which encodes FTN-1 variant with point mutations 555 (E57K and H60G, where the first methionine is counted as 0) abolishing its ferroxidase 556 activity. Note that combining ets-4(rrr16) and ftn-1(syb2550) mutations completely abolished 557 the enhanced cold resistance of ets-4(-) single mutant. Error bars represent SEM. $n=3 ; 261$ 558396 animals were scored per time point. 
Pekec et al. Figure 5

A

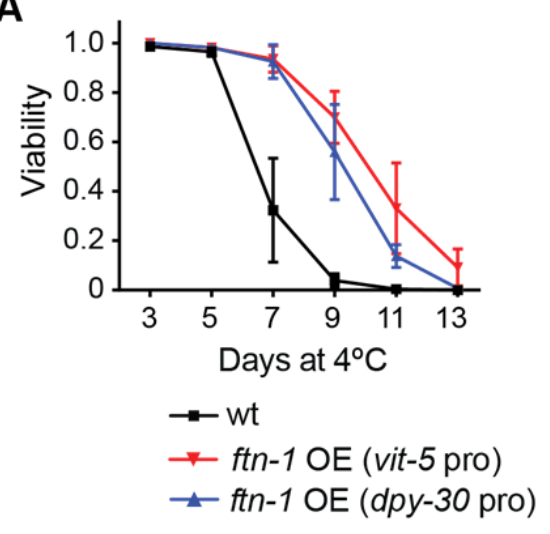

B

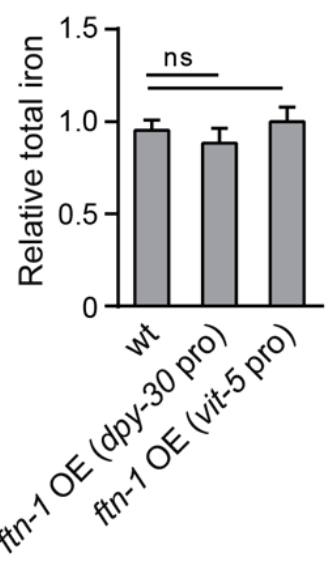

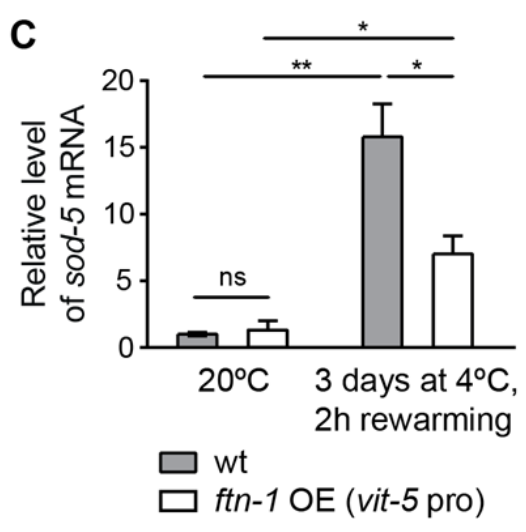

559

560

561 Figure 5. FTN-1 overexpression is sufficient for enhanced cold survival

562 A. Survival of animals of the indicated genotypes subjected to cold, as in $1 \mathrm{~A}$. The ftn-1 563 overexpressing lines (sybSi67 and sybSi72) are marked as ftn-1 OE (dpy-30 pro) and ftn-1

564 OE (vit-5 pro), respectively. Note that both ftn-1-overexpressing strains survived cold better

565 than wt; ftn-1 overexpression from the vit-5 promoter was slightly more beneficial, which is

566 why we chose this strain for additional experiments. Error bars represent SEM, n= 3. 232-

567307 animals were scored per time point.

568 B. Total iron levels, measured by ICP-MS, in C. elegans extracts derived from wild type, or 569 ftn-1 overexpressing, 1 day-old adults, grown at $20^{\circ} \mathrm{C}$. The values were normalized to wild 
570 type. Unpaired two-tailed t-test was used to calculate the $\mathrm{p}$ value, "ns" = not significant. Error

571 bars indicate SEM, $n=3$.

572 C. Relative levels of sod-5 mRNA, measured (by RT-qPCR) in animals of the indicated 573 genotypes, and at the time and temperature. Note that the cold-treated animals were 574 analyzed at around 2 hours into rewarming at $20^{\circ} \mathrm{C}$. mRNA levels were normalized to act-1 575 mRNA. At each time point, the values were then normalized to the wt at $20^{\circ} \mathrm{C}$. Error bars 576 represent SEM. $n=3 ; p$ values were calculated using an unpaired Student t-test. * indicates $577 p<0.05 ;{ }^{* *} p<0.01 ;$ "ns" = not significant. 
Pekec et al. Fig. 6

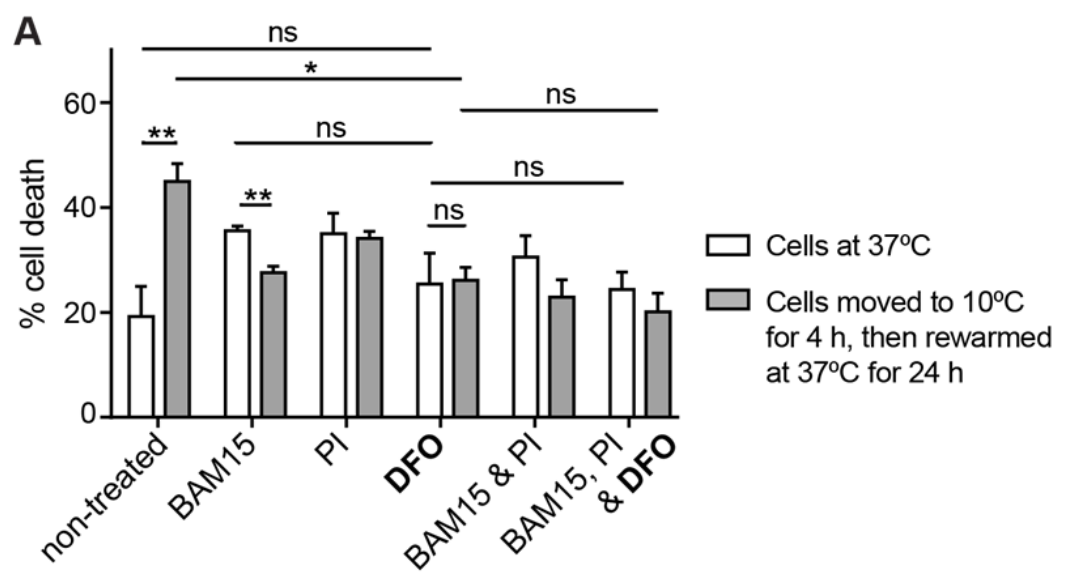

B

NEFH (neurofilament) staining

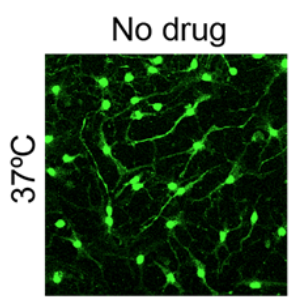

BAM15

DFO
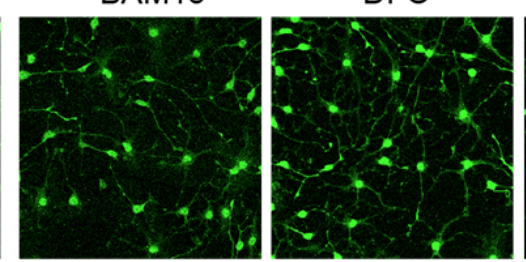

PI
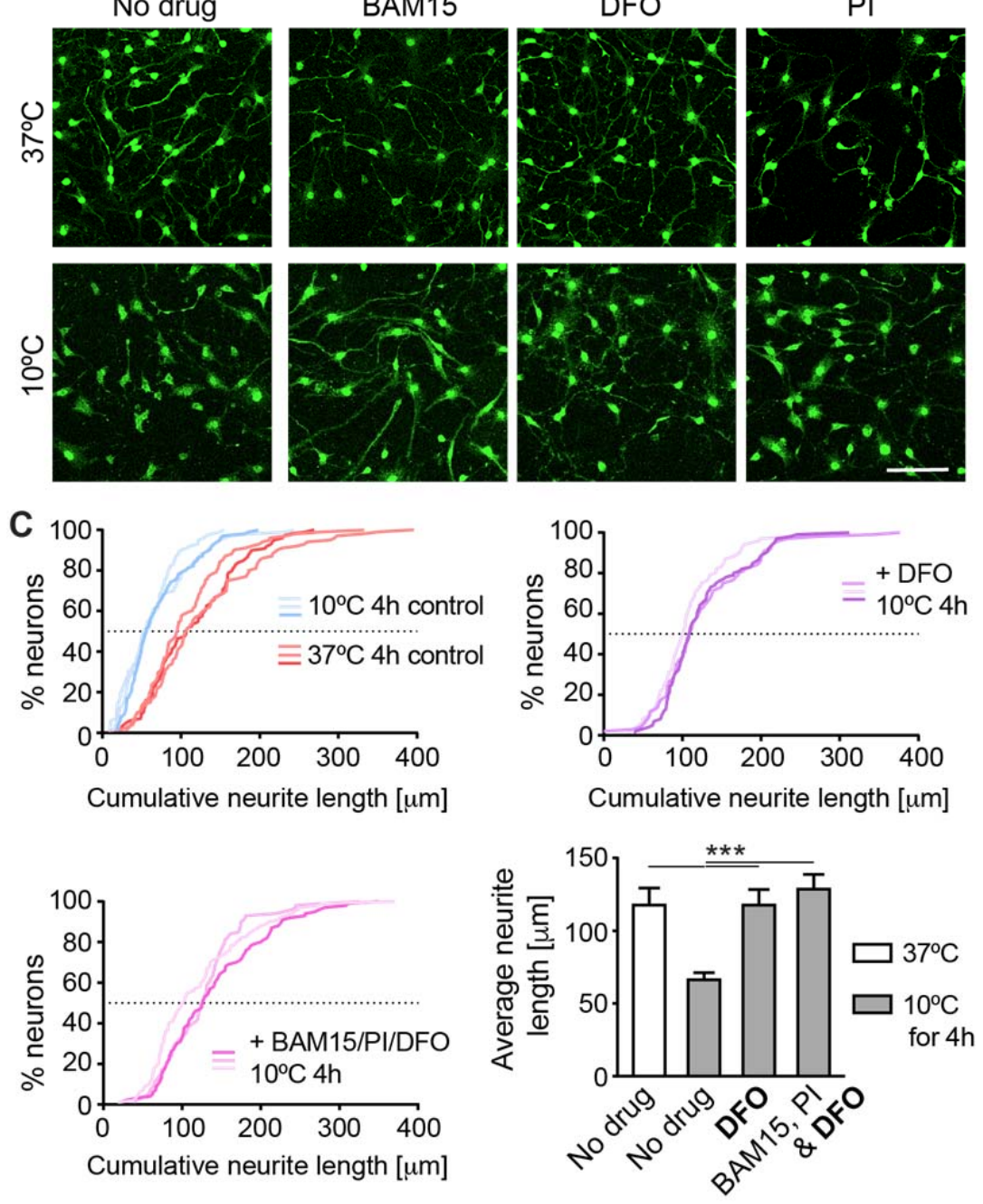
581 A. Viability of murine neurons, subjected to cold and the indicated drugs, was examined by 582 staining with propidium iodide (see Methods for details). "BAM15" is a mitochondrial 583 uncoupling drug, "PI" a cocktail of protease inhibitors, and "DFO" deferoxamine, an iron 584 chelator. Error bars represent SEM. $n=3$ experiments; $p$ values, between cold-treated and 585 control $\left(37^{\circ} \mathrm{C}\right)$ samples, were calculated with Student's t-test, while the ANOVA plus Tukey 586 post hoc test was used to compare samples subjected to different drugs. ${ }^{*}$ indicates $p<$ $5870.05 ;{ }^{* *} \mathrm{p}<0.01$; and "ns" not significant. Note that, in contrast to non-treated cells incubated 588 at $10^{\circ} \mathrm{C}$, treating cells with DFO prevented cell death to a similar extent as the treatment with 589 BAM15, PI, or the combination of drugs.

590 B. Representative confocal images of murine neurons, subjected to cold and the indicated 591 drugs, and immunostained for NEFH to visualize neurites immediately after cold treatment. 592 Scale bar: $40 \mu \mathrm{m}$. Note that the neurites, which in control neurons degenerated upon cold 593 exposure (the bottom left panel), were stabilized by adding DFO, similar to BAM15 or PI.

594 C. Quantifications of neurite lengths, visualized by NEFH labeling, corresponding to B. The 595 cumulative plots (see Methods) compared neurite lengths in cells treated as indicated. Each 596 curve corresponds to one experimental replicate. The bar graph (bottom right) compares 597 average neurite lengths. While cold treatment led to the shortening of neurites, treating cells 598 with DFO alone stabilized the neurites equally well, as when combined with BAM15 and PI. 599 Error bars represent SD. ${ }^{* *}$ indicates $p<0.001$ 
Pekec et al. Fig. 7

A

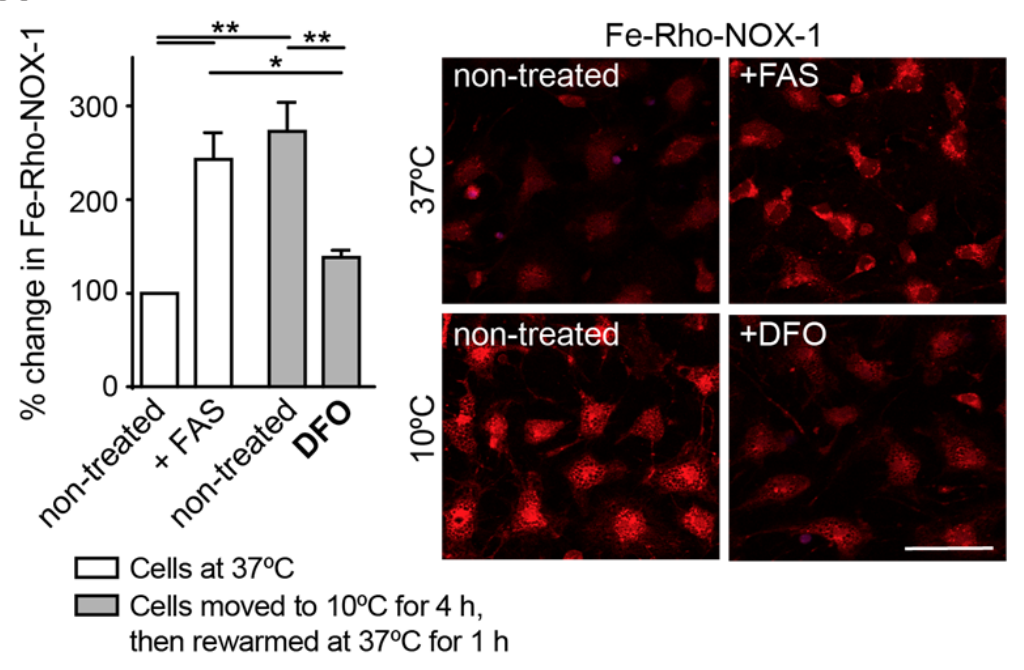

B

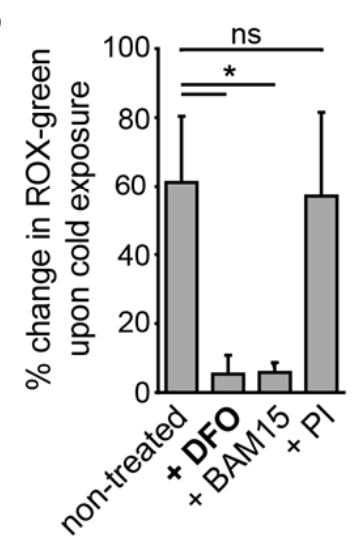

D

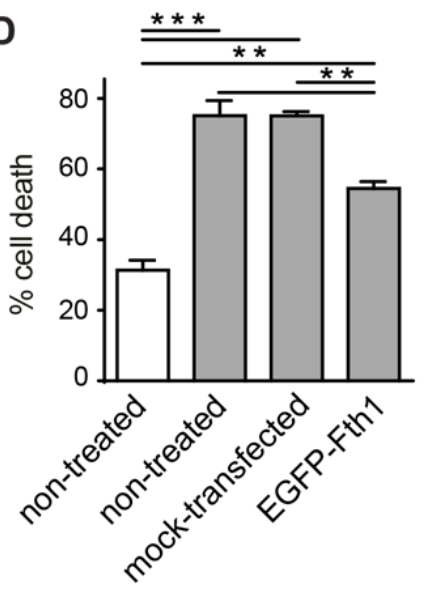

C

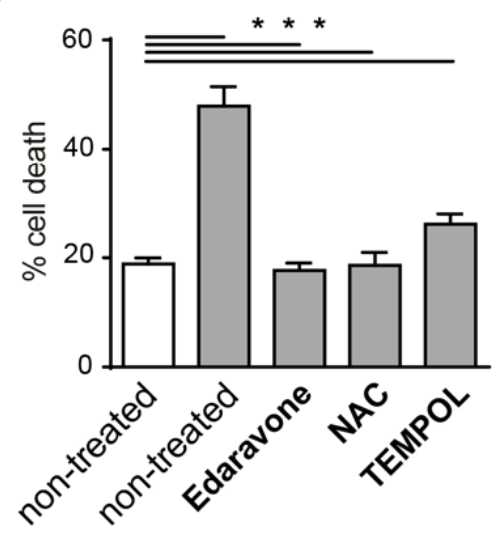

$\square$ Cells at $37^{\circ} \mathrm{C}$

Cells moved to $10^{\circ} \mathrm{C}$ for $4 \mathrm{~h}$ then rewarmed at $37^{\circ} \mathrm{C}$ for $24 \mathrm{~h}$ $\square$ Cells at $37^{\circ} \mathrm{C}$

$\square$ Cells moved to $10^{\circ} \mathrm{C}$ for $4 \mathrm{~h}$, then rewarmed at $37^{\circ} \mathrm{C}$ for $24 \mathrm{~h}$

602 Figure 7. Lowering iron(II) or ROS, or FTH1 overexpression, all enhance neuronal cold 
604 A. Examining iron(II) levels, using the fluorescent FeRhoNox-1 probe (see Methods), in 605 neurons subjected to cold and/or the indicated drugs. Left: quantifications of FeRhoNox-1 606 fluorescence, relative to non-treated cells incubated at $37^{\circ} \mathrm{C}$. "FAS" indicates ammonium 607 ferrous sulfate, here a source of additional iron(II). $n=3 ; p$ values were calculated by ANOVA 608 plus post hoc Tukey test. ${ }^{* *}$ indicates $p<0.01$; and ${ }^{*} p<0.05$. Error bars represent SEM. 609 Representative images are on the right. Scale bar: $50 \mu \mathrm{m}$. Note that the exposure to cold 610 resulted in higher levels of reactive iron, and that this accumulation was prevented by DFO 611 treatment.

612 B. Comparing ROS levels, using the CellROX-green sensor (see Methods), in neurons 613 subjected to cold and the indicated drugs, relative to non-treated cells incubated at $37^{\circ} \mathrm{C} . \mathrm{n}=$ 6143 experiments; $p$ values were calculated by ANOVA plus post hoc Tukey test. * indicates $p<$ 615 0.05; and "ns" not significant. Drugs were administered immediately before transferring cells 616 to cold. Error bars indicate SEM.

617 C. Viability of murine neurons, subjected to cold and the indicated antioxidants, examined by 618 staining with propidium iodide. "Edaravone" acts against oxygen and hydroxyl radicals, and 619 inhibits lipid peroxidation/lipoxygenase pathways; "NAC", N-Acetyl-Cysteine, a glutathione 620 precursor; "TEMPOL" possesses superoxide dismutase (SOD) and catalase (CAT) mimetic 621 properties. Error bars represent SEM. $n=3$ experiments; $p$ values between samples were 622 calculated with ANOVA plus Tukey post hoc test was used to compare samples subjected to 623 different drugs. ${ }^{* * *}$ indicates $p<0.001$. Note that all tested antioxidants enhance cold 624 resistance.

625 D. Viability of murine neurons overexpressing Fth1/ferritin heavy chain. Following lentiviral 626 incorporation of EGFP-fused Fth1, and a 4 hour incubation in the cold, the survival of 627 transfected neurons was compared to controls; the "mock" neurons were transfected with an 628 "empty" pLJM1-EGFP plasmid. Error bars represent SEM. $n=3$ experiments; $p$ values 629 between samples were calculated with ANOVA plus Tukey post hoc test was used to 
bioRxiv preprint doi: https://doi.org/10.1101/2021.01.30.428937; this version posted February 1, 2021. The copyright holder for this preprint (which was not certified by peer review) is the author/funder. All rights reserved. No reuse allowed without permission.

630 compare samples subjected to different drugs. ${ }^{* * *}$ indicates $p<0.001$; and ${ }^{* *} p<0.01$. Note

631 that neurons overexpressing EGFP-Fth1 survive cold better than control neurons.

632 


\section{METHODS}

\section{C. elegans handling and genetic manipulation}

636 Animals were grown at $20^{\circ} \mathrm{C}$ on standard NGM plates, fed with the OP50 E. coli bacteria ${ }^{65}$.

637 All strains used in this study are listed in Table S1. The CRISPR/Cas9 genome editing was

638 used by SunyBiotech to generate the $f t n-1$ ferroxidase-dead mutant (allele syb2550), and to

639 tag daf-16 and pqm-1 (alleles syb707 and syb432, respectively). The latter was achieved 640 through the C-terminal, in-frame insertion of GFP-FLAG (daf-16) or mCHERRY-MYC (pqm-

641 1). The FTN-1 overexpressing strains (alleles sybSi67 and sybSi72) were generated (by

642 SunyBiotech) using the MosSCI method, utilizing the insertion locus ttTi5605. The ftn-1 OE 643 constructs were generated using the MultiSite Gateway Technology, and contain circa $2 \mathrm{~kb}$ 644 of $d p y-30$, or $1.4 \mathrm{~kb}$ of vit-5 promoter, the genomic ftn-1 DNA, and $0.7 \mathrm{~kb}$ of the unc-54 645 3'UTR. The sod-5::GFP strain, GA411, was kindly provided by David Gems.

646 For RNAi experiments, $1 \mathrm{mM}$ IPTG was added to an overnight culture of RNAi 647 bacteria. $300 \mu \mathrm{l}$ of bacterial suspension was plated onto agar plates containing $100 \mu \mathrm{l} / \mathrm{ml}$ 648 Carbenicillin and $1 \mathrm{mM}$ IPTG. The L4440 (empty) vector was used as a negative RNAi 649 control. Animals were typically placed on RNAi plates as L1 larvae, and then were grown to 650 day 1 adulthood at $20^{\circ} \mathrm{C}$, at which time point they were cold-adapted and scored as 651 described. The RNAi clones used in this study came from either Ahringer or Vidal libraries.

\section{The assay for C. elegans cold survival}

654 Unless stated otherwise, all cold survival experiments were performed in the following way: 655 prior to cold adaptation, animals were grown at $20^{\circ} \mathrm{C}$ for two generations on OP50. They 656 were then synchronized by bleaching, and L1 larvae were grown until day 1 of adulthood at $65720^{\circ} \mathrm{C}$. At day 1 of adulthood, they were cold-adapted at $10^{\circ} \mathrm{C}$ for $2 \mathrm{~h}$, and then shifted to $4^{\circ} \mathrm{C}$. 
658 Animals were sampled at indicated intervals, and their survival was scored after $24 \mathrm{~h}$

659 recovery at $20^{\circ} \mathrm{C}$. Pairwise Wilcoxon signed rank test, in $\mathrm{R}$, was used

660 for statistical comparisons of survival curves between strains. Original counting data and 661 statistical results are included in Table S2.

662 To examine the sensitivity to ferric ammonium citrate (FAC), animals were grown at $66320^{\circ} \mathrm{C}$, from L1s to day 1 of adulthood, on different concentrations of FAC, which was added 664 to agar in plates. Animals were then cold-treated and scored for survival as above.

Poly-A mRNA sequencing

6671000 day one adult animals were collected $24 \mathrm{~h}$ after cold adaptation. All steps up to Trizol 668 collection were performed at $4^{\circ} \mathrm{C}$. Animals were washed 2 times in M9 buffer and snap669 frozen in Trizol. Samples were then lysed by freeze/thaw cycles, and RNA extraction 670 proceeded as described before ${ }^{66}$. Genomic DNA was removed using RNeasy Plus Mini Kit 671 (Qiagen). Quality of RNA was monitored by Bioanalyzer RNA Nano chip (Agilent 672 Technologies). The library was prepared using the TruSeq Library Preparation Kit (llumina). 673 Poly-A mRNA was sequenced using a Hiseq 50-cycle single-end reads protocol on a HiSeq 6742500 device (Illumina). Raw RNA sequence data were deposited at GEO with accession No. 675 GSE131870.

678 Genomic data analysis

679 FASTQC ${ }^{67}$ was used to check the quality of the raw sequence data. The reads were 680 mapped to the C. elegans genome (Ensembl WBcel235) using STAR ${ }^{68}$, with default 681 parameters except: outFilterMismatchNmax 3,outFilterMultimapNmax 1, alignIntronMax 682 15000, outFilterScoreMinOverLread 0.33, outFilterMatchNminOverLread 0.33 . Count 
683 matrices were generated for the number of reads overlapping with the exons of protein-

684 coding genes using summarizeOverlaps from GenomicFeatures ${ }^{69}$. Gene expression levels

685 (exonic) from RNA-seq data were quantified as described previously ${ }^{70}$. After normalization

686 for library size, log2 expression levels were calculated after adding a pseudocount of $8(y=$

$687 \log 2[x+8])$. Genes with 2-fold changes in both replicates were considered significantly

688 differentially expressed. The ChIP bigWig files for PQM-1 and DAF-16 was obtained from

689 ENCODE project ${ }^{30}$. EnrichedHeatmap ${ }^{71}$ was used to generate the integrative heatmap.

\section{RT-qPCR}

692 Around 1000, 1 day-old adult C. elegans were collected at $20^{\circ} \mathrm{C}$ prior to cold adaptation, or 693 at 1 day/ 3 days at $4^{\circ} \mathrm{C}$ after adaptation, washed 2 times in M9 buffer at the respective 694 temperature, and flash-frozen in Trizol. RNA was isolated as above. $300 \mathrm{ng}$, or $1000 \mathrm{ng}$ of 695 RNA was used to prepare cDNA with the QuantiTect Reverse Transcription kit (Quiagen), or 696 High-Capacity cDNA Reverse Transcription Kit (Applied Biosystems). cDNA was diluted

$697 \quad 1: 10$ or $1: 5$ and $5 \mu \mathrm{l}$ or $2 \mu \mathrm{l}$ was used with the Light Cycler Syber Green master mix (Roche), 698 or AMPLIFY ME SG Universal Mix (Blirt), and Ct values were calculated using Light Cycler 699480 (Roche). act-1 (actin) was used as the reference gene. Statistical analysis on all of the

700 experiments was performed using the GraphPad/ Prism 8. Statistical method used to 701 calculate $\mathrm{P}$ value is indicated in the figure legend. The following primers were used: act-1 702 FW: CTATGTTCCAGCCATCCTTCTTGG, act-1 RV: TGATCTTGATCTTCATGGTTGATGG; 703 ets-4 FW: CTGAGAACCCGAATCATCCA, ets-4 RV: TCATTCATGTCTTGACTGCTCC; ftn-1

704 FW: CGGCCGTCAATAAACAGATTAACG, ftn-1 RV: CACGCTCCTCATCCGATTGC; daf-16 705 FW: AAAGAGCTCGTGGTGGGTTA, daf-16 RV: TTCGAGTTGAGCTTTGTAGTCG; pqm-1 706 FW: GTGCATCCACAGTAAACCTAATG, pqm-1 RV: ATTGCAGGGTTCAGATGGAG; ftn-2 707 FW: GAGCAGGTCAAATCTATCAACG, ftn-2 RV: TCGAAGACGTACTCTCCAACTC; sod-5 708 FW: ATTGCCAATGCCGTTCTTCC, sod-5 RV: AGCCAAACAGTTCCGAAGAC. 
710 Fluorescent imaging of C. elegans intestinal nuclei

7111 day-old $C$. elegans were anesthetized in $20 \mu \mathrm{M}$ levamisol and placed on $2 \%$ agar pads.

712 DAF-16::GFP:::FLAG and PQM-1::mCHERRY::MYC were imaged on a spinning disc

713 confocal microscope: Zeiss Axiolmager equipped with a Yokogawa CSU-W1 scan-head, 2

714 PCO Edge cameras, a Plan-Apochromat 40x/1.3 oil objective and two $488 \mathrm{~nm}$ and $561 \mathrm{~nm}$

715 laser lines. Laser intensities and exposure times were kept constant for all samples, camera

716 binning was set to 2 . Mean fluorescence intensity in intestinal cell nuclei (three per

717 nematode) was quantified manually with FIJI/ImageJ ${ }^{72}$. The mean fluorescence intensities

718 of each nucleus were averaged and represent one data point for each animal. 10-15 animals

719 were scored per genotype and biological replicate, in total around 40 animals per condition.

720 Statistical analysis was performed using the GraphPad/ Prism 8. Two-tailed, unpaired, t-test

721 was performed to calculate the $p$ value between conditions.

722

723 Fluorescent imaging of SOD-5::GFP

7241 day-old adults were anesthetized in $10 \mathrm{mM}$ levamisol and placed on $2 \%$ agar pads. The

725 GFP fluorescence was imaged on Axio Imager.Z2 (Carl Zeiss), equipped with Axiocam 506

726 mono digital camera (Carl Zeiss), and a Plan-Apochromat 63x/1.40 Oil DIC M27 objective.

727 Images, acquired with the same camera settings, were processed with ZEN 2.5 (blue

728 edition) microscope software in an identical manner, and imported into Adobe Illustrator. 10-

72915 animals were imaged per time point and biological replicate.

730

731 Oil red 0 staining and analysis

732 Oil red $\mathrm{O}$ staining was performed as published ${ }^{73}$. In brief, $0.5 \mathrm{~g}$ of Oil Red O powder was

733 mixed in $100 \mathrm{ml}$ isopropanol for $24 \mathrm{~h}$, protected from direct light. This solution was diluted in 
734 water to $60 \%$, stirred $\mathrm{O} / \mathrm{N}$, and sterile-filtered using a $0.22 \mu \mathrm{m}$ pore filter. Between $200-300$

735 day one-old animals were collected with $1 \mathrm{ml}$ of M9 buffer and were washed once with M9.

736 They were fixed in $75 \%$ isopropanol for $15 \mathrm{~min}$ with gentle inversions every 3-4 minutes. 1

$737 \mathrm{ml}$ of filtered $60 \%$ ORO was added to the animals after the removal of isopropanol. Staining

738 was performed for $6 \mathrm{~h}$ on a shaker with maximum speed, covered with aluminum foil.

739 Stained animals were placed on $2 \%$ agar pads and imaged. Imaging and image analysis

740 were performed as described before ${ }^{17}$. Briefly, animals were imaged using a wide-field

741 microscope Z1 (Carl Zeiss) using a 10x objective and a color camera AxioCam MRc (Carl

742 Zeiss). RGB images were first corrected for shading in Zen Blue software (Carl Zeiss).

743 Afterwards, images were analyzed using Fiji/lmageJ software suite ${ }^{72}$, stitched with the

744 Grid/Collection stitching plug-in ${ }^{72}$, and corrected for white balance. After conversion from

745 RGB to HSB color space, red pixels were selected by color thresholding. A binary mask was

746 created with the Saturation channel and applied to the thresholded image. After conversion

747 to 32-bit, zero pixel values were replaced by NaN. The mean intensity of all remaining pixels

748 was used as a representation of the amount of red staining in the animals (Fiji/lmageJ macro

749 available upon request). 10-15 animals were imaged per genotype and biological replicate. 2

750 tailed t-test was used to assess significance with Graph Pad/Prism 8.

751

\section{C. elegans extract preparation}

753 Animals were collected in M9 buffer in a cold room, washed and resuspended in TBS pH 8.0

754 (2000 worms in $50 \mu$ l total volume) with proteinase inhibitors (EDTA-free, Roche) in protein

755 LoBind tubes (Eppendorf). Probes were then homogenized at $4^{\circ} \mathrm{C}$ in Bioruptor Pico

756 sonicator (Diagenode), using 30 sonication cycles (30 s on/off). After lysis confirmation, by

757 microscopic inspection, probes were centrifuged for $2 \mathrm{~h}$ at $21130 \mathrm{~g}$ at $4^{\circ} \mathrm{C}$, and supernatants

758 were transferred to fresh LoBind tubes. Total protein concentration in the soluble fraction

759 was determined by UV absorbance (NanoDrop, Thermo Fisher Scientific). For ICP-MS 
760 analysis, C. elegans extracts were diluted to $10 \mu \mathrm{g} / \mu \mathrm{l}$ concentration and transferred to $2 \mathrm{ml}$

761 glass vials (ALWSCI technologies) with $50 \mu$ glass inserts with bottom spring (Supelco) and 762 kept at $4^{\circ} \mathrm{C}$ before the analysis.

\section{Size exclusion chromatography inductively coupled plasma mass spectrometry}

765 All experiments were performed using a ICP-MS-2030 Inductively Coupled Plasma Mass

766 Spectrometer (Shimadzu, Japan), directly coupled to a Prominence LC 20Ai inert system 767 (Shimadzu, Japan) ${ }^{74}$. Time-Resolved Measurement (TRM) software for LC-ICP-MS was 768 used for controlling both ICP and LC analytical systems. The ICP-MS operates at $1000 \mathrm{~W}$, 769 with an $8.0 \mathrm{ml} \mathrm{min}^{-1}$ argon plasma gas flow, a $0.7 \mathrm{ml} \mathrm{min}^{-1}$ Ar carrier gas flow, and a $1.0 \mathrm{ml}$ $770 \mathrm{~min}^{-1}$ Ar auxiliary gas flow. The sampling depth was $5.0 \mathrm{~mm}$, and the chamber temp. was 771 set to $3^{\circ} \mathrm{C}$. Optimized conditions of the collision cell were $-90 \mathrm{~V}$ of cell gas voltage, $6.5 \mathrm{~V}$ of 772 energy filter voltage, and a $9.0 \mathrm{ml} \mathrm{min}^{-1}$ cell gas $(\mathrm{He})$ flow rate. The separation was 773 performed using BioSEC-5, 300A, $5 \mu \mathrm{m}, 4.6 \times 300 \mathrm{~mm}$ (Agilent, USA) column using $200 \mathrm{mM}$ 774 ammonium nitrate (99.999\% trace metal basis, Sigma Aldrich) pH 8.0 (adjusted by $\mathrm{NH}_{4} \mathrm{OH}$, 775 Sigma Aldrich, Merck group, Poland) as the mobile phase with a flow rate $0.4 \mathrm{ml} \mathrm{min}^{-1}$ and 776 run time $10 \mathrm{~min}$. In all measurements, a $10 \mu \mathrm{l}$ sample loop was used. For iron content 777 quantification in fractions after separation, ferritin (iron) from equine spleen - Type I 778 standard (Sigma Aldrich) was diluted to $100,250,500,1000$ and $2000 \mu \mathrm{g} \mathrm{L}^{-1}$ total metal 779 concentration in mobile phase solution and used to create a standard calibration curve. Iron 780 content in each fraction was normalized to peak area on the chromatogram. Total iron 781 concentration was determined by direct sample injection (LC-ICP-MS) and quantification 782 based on iron standard solution (Sigma Aldrich). The standard calibration curve was created 783 using the same iron concentrations as for SEC-ICP-MS method. 
786 Entire heads of fetal mouse (C57BL/6; gestation day between E9-11) were isolated and the

787 tissue was fragmented into pieces followed by incubation in Trypsin-EDTA (0.05 \%) (Thermo

788 Fisher Scientific, Waltham, USA) for $15 \mathrm{~min}$ at $37^{\circ} \mathrm{C}$. The tissue was subsequently 789 transferred to DMEM/10 \% FCS and triturated by pipetting up and down into single-cell 790 suspension. The cell suspension was transferred on adherent, uncoated tissue culture 791 plates. After the 3 hour incubation in $5 \% \mathrm{CO}^{2}$ at $37^{\circ} \mathrm{C}$, the residual differentiated and non792 neuronal cells readily attached to the bottom of the plate and the floating neuronal stem cells 793 were collected. The neuronal cells were transferred on to low-adhesive 6-well plates, coated 794 with Poly-HEMA (Poly 2-hydroxyethyl methacrylate; Sigma-Aldrich, St. Louis, USA) using 795 DMEM medium (Thermo Fisher Scientific), supplemented with F-12 (Thermo Fisher 796 Scientific), B-27 (Thermo Fisher Scientific), $100 \mathrm{ng} / \mathrm{ml}$ basic fibroblast growth factor (FGF-2, 797 ORF Genetis, Kopavogur, Iceland), $100 \mathrm{ng} / \mathrm{ml}$ epidermal growth factor (EGF, ORF Genetis) 798 and $5 \mu \mathrm{g} / \mathrm{ml}$ heparin (Sigma-Aldrich). After 1 day in culture $\left(5 \% \mathrm{CO}_{2} / 5 \% \mathrm{O}_{2}\right)$, the neuronal 799 cells formed neuronal spheres, which were further cultured and passaged weekly using 800 tissue chopper ${ }^{75}$.

801

\section{Differentiation of NSC to noradrenergic neurons}

803 Neuronal spheres were differentiated towards noradrenergic neurons using available 804 protocols ${ }^{76}$. The spheres were dissociated by chopping into small cell aggregates and 805 plated onto glass coverslips coated with $0.05 \mathrm{mg} / \mathrm{ml}$ poly-D-lysine (Thermo Fisher Scientific) 806 and $3.3 \mu \mathrm{g} / \mathrm{ml}$ laminin (Sigma-Aldrich). Cells were incubated for 5 days in Neurobasal 807 Medium, supplemented with B-27 serum-free supplement, penicillin/streptomycin (all 808 Thermo Fisher Scientific), and neurotrophic factors: $50 \mathrm{ng} / \mathrm{ml}$ BDNF, $30 \mathrm{ng} / \mathrm{ml}$ GDNF 809 (Peprotech, UK), according to a modified protocol described elsewhere ${ }^{76}$. 
812 Neuronal stem cell and noradrenergic neuronal cell identities were confirmed by PCR-based

813 detection of neuronal stem cell (NSC) gene markers: Sox2, Gbx2, Cd-81, Cdh1, S100b,

814 Dach1, Pax6, Olig1, or neural differentiation markers: Cspg4, D $\beta H$, Darpp32, Nestin (NES).

815 Moreover the neuronal spheres were immunostained for neuronal stem cell markers: Nestin

816 (1: 500; DSHB, lowa, USA; ${ }^{77}$, Foxg-1 (1:100; Abcam, Cambridge, UK), Emx1 (1: 100;

817 Millipore, Burlington, USA) and Emx2 (1:100; Abgent, San Diego, USA) and differentiated

818 neurons for Th (1:100; Abcam), S100b (1:100; Abcam), D $3 H$ (1:500; Abcam), Darpp32

819 (1:50; Abcam).

820

821 Subcloning, lentivirus generation and transduction for Fth1 overexpression in mouse 822 noradrenergic-like neurons

823 The mouse Fth1 was introduced into neurons using lentiviral pLJM1 vector for EGFP fusion. 824 pLJM1-EGFP was a gift from David Sabatini (Addgene plasmid \# 19319; 825 http://n2t.net/addgene:19319; RRID:Addgene_19319) ${ }^{78}$. It co-expresses EGFP and a 826 puromycin resistance, and allows for the visualization and selection of transductants carrying 827 N-terminally EGFP-tagged Fth1. After RNA isolation from mouse brain tissue, the cDNA 828 template was synthesized using NEBNext Second Strand Synthesis Enzyme Mix for ds 829 cDNA and Phusion ${ }^{\circledR}$ High-Fidelity DNA Polymerase (M0530, NEB). For constitutive 830 expression, the coding sequence of Fth1 was PCR-amplified (using ProtoScript II

831 Reaction/Enzyme Mix by New England BioLabs, Ipswich, USA).

832 The following primers with specific Gibson's overhangs were used:

833 mFth1overexpGibson_F

834 (TCCGGACTCAGATCTCGAGCTCAAGCTTCGATGACCACCGCGTCTCCCTCG),

835 mFth1overexpGibson_R

836 (GATGAATACTGCCATTTGTCTCGAGGTCGAGTTAGCTCTCATCACCGTGTCCC), and 837 cloned into EcoRI-digested lentiviral pLJM1::EGFP vector. Cloning and DNA preparations 
838 were done using $\mathrm{NEB} \circledast$ Stable Competent E. coli $(\mathrm{C} 3040 \mathrm{H})$, according to Gibson

839 Assembly® protocol (NEB).

840 Lentiviral particles were assembled using a third-generation packaging system. The 841 plasmid pLJM1::EGFP::Fth1 or "empty" pLJM1::EGFP vector, pMDL, pMD2.G and 842 pRSV/REV were mixed (3:2:1:0.8), and human embryonic kidney 293 cells (HEK 293T) $(3 \times$ $84310^{6}$ cells seeded on T75 flask one day before) were transfected using a calcium phosphate 844 protocol. Pseudoviral particles in neuronal maintenance medium were collected at 48 and 72 $845 \mathrm{~h}$ post-transfection and filtered through a $45 \mu \mathrm{m}$ filter. The aliquots were snap-frozen and 846 stored at $-80^{\circ} \mathrm{C}$. Transduction in neurons was done by replacing culture medium with one 847 enriched in lentiviral particles (pLJM1::EGFP::Fth1 or empty pLJM1::EGFP), collected before 848 and supplemented with polybrene $(5 \mu \mathrm{g} / \mathrm{mL})$. After $24 \mathrm{~h}$ incubation, medium was discarded 849 and a new one with lentiviral vector was added for subsequent $24 \mathrm{~h}$. At $48 \mathrm{~h}$ after 850 transduction, the medium was replaced with fresh viral-free medium and, at $96 \mathrm{~h}$ post851 transduction, selection with puromycin was initiated for further 48 hours.

853 Cold treatment of noradrenergic-like neurons

854 The assay was established using two independent humidified airtight cell culture incubators.

855 One water-jacketed type incubator was additionally equipped with cooler unit $\left(10^{\circ} \mathrm{C}\right)$ and the 856 other incubator was set to $37^{\circ} \mathrm{C}$. Both contained atmosphere control, which was set to $5 \%$ $857 \mathrm{CO}_{2} / 5 \% \mathrm{O}_{2}$. If not stated otherwise, differentiated neuronal cultures were placed in a $10^{\circ} \mathrm{C}$ 858 incubator for 4 hours and then returned to the $37^{\circ} \mathrm{C}$-incubator for additional 24 hours of 859 rewarming. Such cooling/rewarming paradigm demonstrated a statistically relevant rise of 860 cell death as early as $4 \mathrm{~h}$ into cooling, which was used in subsequent assays. To evaluate 861 neuroprotective effects of compounds, neuronal culture medium was replaced with a 862 Neurobasal medium, without neurotrophic factors supplemented with $100 \mu \mathrm{M}$ deferoxamine 863 (DFO concentration determined based on dose curve at $10^{\circ} \mathrm{C}$ ) (Sigma-Aldrich), $100 \mathrm{nM}$ 
864 BAM15 (Tocris, Bristol, UK), 1:500 dilution of protease inhibitor cocktail III PI (Sigma865 Aldrich). All compounds were provided as a single or as a combined treatment. The effects 866 of antioxidants were tested by supplementation of neuronal maintenance medium with 50 $867 \mu \mathrm{M}$ Edaravone (Sigma-Aldrich), $50 \mu \mathrm{M}$ TEMPOL (Sigma-Aldrich), or $10 \mu \mathrm{M} \mathrm{N}-A$ cetyl-L868 cysteine NAC (Sigma-Aldrich), following procedures described above. Drugs concentration 869 was determined based on dose curves at $10^{\circ} \mathrm{C}$.

870

$871 \quad$ Propidium iodide staining

872 After 4 hours of cooling at $10^{\circ} \mathrm{C}$, and additional 24 hours of rewarming at $37^{\circ} \mathrm{C}$, neurons 873 cultured on glass coverslips were incubated in the presence of $10 \mu \mathrm{g} / \mathrm{ml}$ propidium iodide 874 (PI) (Cayman Chemical, Ann Arbor, USA) diluted in phosphate-buffered saline (PBS), and 875 costained with $1 \mu \mathrm{g} / \mathrm{ml}$ Hoechst 33342 (Life Technologies) for $25 \mathrm{~min}$ at $37^{\circ} \mathrm{C}$. Cells were 876 then fixed in ice-cold $4 \%$ buffered formaldehyde for $15 \mathrm{~min}$, washed twice in PBS and 877 placed in histology mounting medium (Sigma-Aldrich) on a glass slide. The prepared 878 material was imaged using fluorescence microscope (Leica DMI 4000B, Germany) and LAS 879 X SP8 software. Counting of total cells (blue nuclei) and necrotic cells (red-PI positive and 880 round) was performed on 2-3 images from 3 coverslips as replicates. Collected data were 881 statistically analyzed using Prism software (version 6.01 for Windows, La Jolla, CA).

882

883 CellROX-green staining of ROS production

884 To demonstrate whether cold-stabilizing drugs inhibit intracellular ROS levels, neurosphere885 derived neurons were incubated with cell-permeable dye, CellROX Green Reagent (Life 886 Technologies, Carlsbad, USA), at the final concentration of $5 \mu \mathrm{M}$, according to the procedure 887 described elsewhere ${ }^{46}$. Green fluorescence was emitted after dye binding to DNA, only 888 upon its oxidation. In brief, murine neuronal cells were differentiated in 24-well plates on 889 glass coverslips. Control cells (group 1) were maintained only at $37^{\circ} \mathrm{C}$ (non-cold control). 
890 Other cells (group 2) were exposed for $4 \mathrm{hrs}$ to $10^{\circ} \mathrm{C}$, in the presence or absence of the

891 following drugs: $100 \mu \mathrm{M}$ DFO, $100 \mathrm{nM}$ BAM15 and 1:500 dilution of protease inhibitor

892 cocktail (PI). Subsequently, after 5 min rewarming at room temperature on the bench, ROS

893 accumulation in neurons was assessed for both groups, by staining with fluorogenic Cell-

894 ROX green reagent in the dark for $30 \mathrm{~min}$ at $37^{\circ} \mathrm{C}$. Additionally, before cooling, reference

895 neurons (for time 0) were labeled to detect fluorescent signal in initial precooling cultures. Z-

896 stack well-focused confocal images at $0.55 \mu \mathrm{m}$ intervals in the z-axis of 4 culture areas per

897 each treatment and condition were collected for both groups. Maximal intensity projections

898 of the $Z$ stack images were produced for data analysis. Microscopy images were taken using

899 a Leica TCS SP5 confocal microscope and LAS X SP8 software. The change in ROS

900 production at $10^{\circ} \mathrm{C}$ incubated cells vs cells maintained only in $37^{\circ} \mathrm{C}$ was calculated using the

901 following formula: $\left(F_{2}-F_{1}\right) / F_{0} \times 100 \%$. The CellROX-green fluorescence of non-cold control

902 cells $\left(\mathrm{F}_{1}\right.$; mean intensity) was subtracted from CellROX-fluorescence at the end of cold

903 treatment $\left(F_{2} ;\right.$ mean intensity). While $F_{0}$ stands for the initial mean CellROX-green

904 fluorescent intensity of culture areas before $10^{\circ} \mathrm{C}$ cooling.

905

906 Determination of iron(II) with FeRhoNox-1

907 Intracellular iron levels were measured according to the manufacturer's protocol. Cells were

908 cultured in a glass-bottom dish, and exposed to indicated agents in the cold. Next, cells were

909 rinsed twice with HBSS, then $5 \mu \mathrm{M}$ FeRhoNox ${ }^{\mathrm{TM}}-1$ solution (Goryo Chemical, Inc., Sapporo,

910 Japan) was added and incubated in the dark at $37^{\circ} \mathrm{C}$ for $1 \mathrm{~h}$, and then washed twice with

911 HBSS. To track changes in $\mathrm{Fe}^{2+}$ over time, after cooling neurons at 10 degrees, the probe

912 signal was recorded at 1, 4 and 8 hours of rewarming. In turn, to examine iron(II) right after

913 cooling (0 hour), incubation with the reagent was completed at the end of the 4-hour cold

914 exposure. The FeRhoNox signal was visualized using a confocal microscope (Leica TCS

915 SP5, Germany) and LAS X SP8 software. FeRhoNox-1 was excited at $543 \mathrm{~nm}$ and 
916 measured at $570 \mathrm{~nm}$. Fluorescence intensity of Z-stacked confocal images of neuronal

917 culture (maximal intensity projection of 7 image z-stacks at $0.55 \mu \mathrm{m}$ intervals in the z-axis)

918 was analyzed using ImageJ.

919

$920 \quad$ Neurite tracing

921 Cultured neurons with, or without, DFO or BAM15/PI/DFO were fixed with $4 \%$ 922 paraformaldehyde, permeabilized and washed with $0.1 \%$ Triton X-100 in phosphate923 buffered saline, and stained by antibody against $\mathrm{NEFH}$. NEFH ${ }^{+}$neurite paths were traced

924 with the 'Simple Neurite Tracer' plugin ${ }^{79}$, ImageJ, using Z-stacked confocal images of 925 neuronal culture (maximal intensity projection of 7 image z-stacks at $0.55 \mu \mathrm{m}$ intervals in the 926 z-axis). Cumulative frequency plots of neurite lengths, for each experimental group, were 927 built using GraphPad Prism version 6.01 for Windows, GraphPad Software, La Jolla 928 California USA. 


\section{REFERENCES}

932 1. Melvin, R.G. \& Andrews, M.T. Torpor induction in mammals: recent discoveries

933 fueling new ideas. Trends Endocrinol Metab 20, 490-498 (2009).

934 2. Andrews, M.T. Molecular interactions underpinning the phenotype of hibernation in 935 mammals. J Exp Biol 222 (2019).

$936 \quad 3 . \quad$ Geiser, F. Hibernation. Curr Biol 23, R188-193 (2013).

937 4. Wu, C.W. \& Storey, K.B. Life in the cold: links between mammalian hibernation and $938 \quad$ longevity. Biomol Concepts 7, 41-52 (2016).

939 5. Blanco, M.B., Dausmann, K.H., Faherty, S.L. \& Yoder, A.D. Tropical heterothermy is

6. Cerri, M. The Central Control of Energy Expenditure: Exploiting Torpor for Medical "cool": The expression of daily torpor and hibernation in primates. Evol Anthropol 27, 147-161 (2018).

952

7. Nordeen, C.A. \& Martin, S.L. Engineering Human Stasis for Long-Duration Applications. Annu Rev Physiol 79, 167-186 (2017).

Spaceflight. Physiology (Bethesda) 34, 101-111 (2019).

8. Yenari, M.A. \& Han, H.S. Neuroprotective mechanisms of hypothermia in brain ischaemia. Nat Rev Neurosci 13, 267-278 (2012).

9. Kutcher, M.E., Forsythe, R.M. \& Tisherman, S.A. Emergency preservation and resuscitation for cardiac arrest from trauma. Int J Surg 33, 209-212 (2016).

10. Loeb, J. \& Northrop, J.H. Is There a Temperature Coefficient for the Duration of Life? Proc Natl Acad Sci U S A 2, 456-457 (1916).

11. Conti, B. et al. Transgenic mice with a reduced core body temperature have an increased life span. Science 314, 825-828 (2006).

955

956

957

958

959

12. Frezal, L. \& Felix, M.A. C. elegans outside the Petri dish. Elife 4 (2015).

13. Klass, M.R. Aging in the nematode Caenorhabditis elegans: major biological and environmental factors influencing life span. Mech Ageing Dev 6, 413-429 (1977).

14. Xiao, R. et al. A Genetic Program Promotes C. elegans Longevity at Cold Temperatures via a Thermosensitive TRP Channel. Cell 152, 806-817 (2013).

15. Ohta, A., Ujisawa, T., Sonoda, S. \& Kuhara, A. Light and pheromone-sensing neurons regulates cold habituation through insulin signalling in Caenorhabditis elegans. Nat Commun 5 (2014).

964 17. Habacher, C. et al. Ribonuclease-Mediated Control of Body Fat. Dev Cell 39, 359965

16. Robinson, J.D. \& Powell, J.R. Long-term recovery from acute cold shock in Caenorhabditis elegans. BMC Cell Biol 17, 2 (2016). 369 (2016). 
966

967

968

969

970

971

972

973

974

975

976

977

978

979

980

981

982

983

984

985

986

987

988

989

990

991

992

993

994

995

996

997

998

999

1000

1001

1002

1003

18. Murray, P., Hayward, S.A., Govan, G.G., Gracey, A.Y. \& Cossins, A.R. An explicit test of the phospholipid saturation hypothesis of acquired cold tolerance in Caenorhabditis elegans. Proc Natl Acad Sci U S A 104, 5489-5494 (2007).

19. Savory, F.R., Sait, S.M. \& Hope, I.A. DAF-16 and Delta(9) Desaturase Genes Promote Cold Tolerance in Long-Lived Caenorhabditis elegans age-1 Mutants. Plos One 6 (2011).

20. Habacher, C. \& Ciosk, R. ZC3H12A/MCPIP1/Regnase-1-related endonucleases: An evolutionary perspective on molecular mechanisms and biological functions. Bioessays 39 (2017).

21. Thyagarajan, B. et al. ETS-4 is a transcriptional regulator of life span in Caenorhabditis elegans. PLoS Genet 6, e1001125 (2010).

22. Murphy, C.T. \& Hu, P.J. Insulin/insulin-like growth factor signaling in C. elegans. WormBook, 1-43 (2013).

23. Riddle, D.L., Swanson, M.M. \& Albert, P.S. Interacting genes in nematode dauer larva formation. Nature 290, 668-671 (1981).

24. Ayyadevara, S., Alla, R., Thaden, J.J. \& Reis, R.J.S. Remarkable longevity and stress resistance of nematode PI3K-null mutants. Aging Cell 7, 13-22 (2008).

25. Tepper, R.G. et al. PQM-1 complements DAF-16 as a key transcriptional regulator of DAF-2-mediated development and longevity. Cell 154, 676-690 (2013).

26. Senchuk, M.M. et al. Activation of DAF-16/FOXO by reactive oxygen species contributes to longevity in long-lived mitochondrial mutants in Caenorhabditis elegans. Plos Genetics 14 (2018).

27. Dowen, R.H., Breen, P.C., Tullius, T., Conery, A.L. \& Ruvkun, G. A microRNA program in the $C$. elegans hypodermis couples to intestinal mTORC2/PQM-1 signaling to modulate fat transport. Genes Dev 30, 1515-1528 (2016).

28. Rajan, M. et al. NHR-14 loss of function couples intestinal iron uptake with innate immunity in C. elegans through PQM-1 signaling. Elife 8 (2019).

29. O'Brien, D. et al. A PQM-1-Mediated Response Triggers Transcellular Chaperone Signaling and Regulates Organismal Proteostasis. Cell Rep 23, 3905-3919 (2018).

30. Davis, C.A. et al. The Encyclopedia of DNA elements (ENCODE): data portal update. Nucleic Acids Res 46, D794-D801 (2018).

31. Anderson, C.P. \& Leibold, E.A. Mechanisms of iron metabolism in Caenorhabditis elegans. Front Pharmacol 5 (2014).

32. Muckenthaler, M.U., Rivella, S., Hentze, M.W. \& Galy, B. A Red Carpet for Iron Metabolism. Cell 168, 344-361 (2017).

33. Gourley, B.L., Parker, S.B., Jones, B.J., Zumbrennen, K.B. \& Leibold, E.A. Cytosolic aconitase and ferritin are regulated by iron in Caenorhabditis elegans. $J$ Biol Chem 278, 3227-3234 (2003). 
1004

1005

1006

1007

1008

1009

1010

1011

1012

1013

1014

1015

1016

1017

1018

1019

1020

1021

1022

1023

1024

1025

1026

1027

1028

1029

1030

1031

1032

1033

1034

1035

1036

1037

1038

1039

1040

1041

34. Kim, Y.I., Cho, J.H., Yoo, O.J. \& Ahnn, J. Transcriptional regulation and life-span modulation of cytosolic aconitase and ferritin genes in C. elegans. J Mol Biol 342, 421-433 (2004).

35. Cha'on, U. et al. Disruption of iron homeostasis increases phosphine toxicity in Caenorhabditis elegans. Toxicol Sci 96, 194-201 (2007).

36. Ackerman, D. \& Gems, D. Insulin/IGF-1 and hypoxia signaling act in concert to regulate iron homeostasis in Caenorhabditis elegans. PLoS Genet 8, e1002498 (2012).

37. Consortium, C.e.D.M. large-scale screening for targeted knockouts in the Caenorhabditis elegans genome. G3 (Bethesda) 2, 1415-1425 (2012).

38. James, S.A. et al. Direct in vivo imaging of ferrous iron dyshomeostasis in ageing Caenorhabditis elegans. Chem Sci 6, 2952-2962 (2015).

39. Theil, E.C. Ferritin: The Protein Nanocage and Iron Biomineral in Health and in Disease. Inorg Chem 52, 12223-12233 (2013).

40. Halliwell, B. \& Gutteridge, J.M. Oxygen toxicity, oxygen radicals, transition metals and disease. Biochem J 219, 1-14 (1984).

41. Gutteridge, J.M. \& Halliwell, B. Free radicals and antioxidants in the year 2000. A historical look to the future. Ann N Y Acad Sci 899, 136-147 (2000).

42. Valentini, S. et al. Manipulation of in vivo iron levels can alter resistance to oxidative stress without affecting ageing in the nematode C. elegans. Mechanisms of Ageing and Development 133, 282-290 (2012).

43. Frokjaer-Jensen, C. et al. Single-copy insertion of transgenes in Caenorhabditis elegans. Nat Genet 40, 1375-1383 (2008).

44. Wang, Y., Branicky, R., Noe, A. \& Hekimi, S. Superoxide dismutases: Dual roles in controlling ROS damage and regulating ROS signaling. J Cell Biol 217, 1915-1928 (2018).

45. Doonan, R. et al. Against the oxidative damage theory of aging: superoxide dismutases protect against oxidative stress but have little or no effect on life span in Caenorhabditis elegans. Genes Dev 22, 3236-3241 (2008).

46. Ou, J.X. et al. iPSCs from a Hibernator Provide a Platform for Studying Cold Adaptation and Its Potential Medical Applications. Cell 173, 851-+ (2018).

47. Hudson, J.W. \& Scott, I.M. Daily Torpor in the Laboratory Mouse, Mus-Musculus Var Albino. Physiol Zool 52, 205-218 (1979).

48. Amemiya, S. et al. Anti-apoptotic and neuroprotective effects of edaravone following transient focal ischemia in rats. Eur J Pharmacol 516, 125-130 (2005).

49. Feng, L.D. et al. Efficacy and safety of edaravone for acute intracerebral haemorrhage: protocol for a systematic review and meta-analysis. Bmj Open 10 (2020). 
1042

1043

1044

1045

1046

1047

1048

1049

1050

1051

1052

1053

1054

1055

1056

1057

1058

1059

1060

1061

1062

1063

1064

1065

1066

1067

1068

1069

1070

1071

1072

1073

1074

1075

1076

1077

1078

1079

1080

1081

1082

50. Otomo, E. et al. Effect of a novel free radical scavenger, edaravone (MCl-186), on acute brain infarction - Randomized, placebo-controlled, double-blind study at multicenters. Cerebrovasc Dis 15, 222-229 (2003).

51. Cuzzocrea, S. et al. Beneficial effects of $n$-acetylcysteine on ischaemic brain injury. Brit J Pharmacol 130, 1219-1226 (2000).

52. Ezerina, D., Takano, Y., Hanaoka, K., Urano, Y. \& Dick, T.P. N-Acetyl Cysteine Functions as a Fast-Acting Antioxidant by Triggering Intracellular H2S and Sulfane Sulfur Production. Cell Chem Biol 25, 447-+ (2018).

53. Moss, H.G., Brown, T.R., Wiest, D.B. \& Jenkins, D.D. N-Acetylcysteine rapidly replenishes central nervous system glutathione measured via magnetic resonance spectroscopy in human neonates with hypoxic-ischemic encephalopathy. J Cerebr Blood F Met 38, 950-958 (2018).

54. Mehta, S.H., Webb, R.C., Ergul, A., Tawfik, A. \& Dorrance, A.M. Neuroprotection by tempol in a model of iron-induced oxidative stress in acute ischemic stroke. $A m \mathrm{~J}$ Physiol Regul Integr Comp Physiol 286, R283-288 (2004).

55. Wilcox, C.S. Effects of tempol and redox-cycling nitroxides in models of oxidative stress. Pharmacol Therapeut 126, 119-145 (2010).

56. Biggar, K.K. et al. Modulation of Gene Expression in Key Survival Pathways During Daily Torpor in the Gray Mouse Lemur, Microcebus murinus. Genomics Proteomics Bioinformatics 13, 111-118 (2015).

57. Faherty, S.L., Villanueva-Canas, J.L., Blanco, M.B., Alba, M.M. \& Yoder, A.D. Transcriptomics in the wild: Hibernation physiology in free-ranging dwarf lemurs. Mol Ecol 27, 709-722 (2018).

58. Wu, C.W. \& Storey, K.B. FoxO3a-mediated activation of stress responsive genes during early torpor in a mammalian hibernator. Mol Cell Biochem 390, 185-195 (2014).

59. Huang, H. \& Salahudeen, A.K. Cold induces catalytic iron release of cytochrome P450 origin: a critical step in cold storage-induced renal injury. Am J Transplant 2, 631-639 (2002).

60. Rauen, U. et al. Cold-induced apoptosis of hepatocytes: Mitochondrial permeability transition triggered by nonmitochondrial chelatable iron. Free Radical Bio Med 35, 1664-1678 (2003).

61. Balaban, R.S., Nemoto, S. \& Finkel, T. Mitochondria, oxidants, and aging. Cell 120, 483-495 (2005).

62. Kell, D.B. Iron behaving badly: inappropriate iron chelation as a major contributor to the aetiology of vascular and other progressive inflammatory and degenerative diseases. BMC Med Genomics 2, 2 (2009).

63. Dixon, S.J. \& Stockwell, B.R. The role of iron and reactive oxygen species in cell death. Nat Chem Biol 10, 9-17 (2014).

64. Kelley, L.A., Mezulis, S., Yates, C.M., Wass, M.N. \& Sternberg, M.J. The Phyre2 web portal for protein modeling, prediction and analysis. Nat Protoc 10, 845-858 (2015). 
1083 65. Brenner, S. The genetics of Caenorhabditis elegans. Genetics 77, 71-94 (1974).

1084

1085

1086

1087

1088

1089

1090

1091

1092

1093

1094

1095

1096

1097

1098

1099

1100

1101

1102

1103

1104

1105

1106

1107

1108

1109

1110

1111

1112

1113

1114

1115

1116

1117

1118

66. Arnold, A. et al. Functional characterization of C-elegans Y-box-binding proteins reveals tissue-specific functions and a critical role in the formation of polysomes. Nucleic Acids Res 42, 13353-13369 (2014).

67. Andrews, S. in FASTQC. A quality control tool for high throughput sequence data (http://www.bioinformatics.babraham.ac.uk/projects/fastqc; 2010).

68. Dobin, A. et al. STAR: ultrafast universal RNA-seq aligner. Bioinformatics 29, 15-21 (2013).

69. Lawrence, M. et al. Software for Computing and Annotating Genomic Ranges. Plos Comput Biol 9 (2013).

70. Hendriks, G.J., Gaidatzis, D., Aeschimann, F. \& Grosshans, H. Extensive Oscillatory Gene Expression during C. elegans Larval Development. Mol Cell 53, 380-392 (2014).

71. Gu, Z.G., Eils, R., Schlesner, M. \& Ishaque, N. EnrichedHeatmap: an R/Bioconductor package for comprehensive visualization of genomic signal associations. Bmc Genomics 19 (2018).

72. Schindelin, J. et al. Fiji: an open-source platform for biological-image analysis. Nat Methods 9, 676-682 (2012).

73. O'Rourke, E.J., Conery, A.L. \& Moy, T.I. Whole-animal high-throughput screens: the C. elegans model. Methods Mol Biol 486, 57-75 (2009).

74. Karas, K., Ziola-Frankowska, A. \& Frankowski, M. Chemical Speciation of Aluminum in Wine by LC-ICP-MS. Molecules 25 (2020).

75. Ebert, A.D. et al. EZ spheres: a stable and expandable culture system for the generation of pre-rosette multipotent stem cells from human ESCs and iPSCs. Stem Cell Res 10, 417-427 (2013).

76. Mahabadi, V.P., Movahedin, M., Semnanian, S., Mirnajafi-zadeh, J. \& Faizi, M. In Vitro Differentiation of Neural Stem Cells into Noradrenergic-Like Cells. Int J Mol Cell Med 4, 22-31 (2015).

77. Hockfield, S. \& McKay, R.D. Identification of major cell classes in the developing mammalian nervous system. J Neurosci 5, 3310-3328 (1985).

78. Sancak, Y. et al. The Rag GTPases bind raptor and mediate amino acid signaling to mTORC1. Science 320, 1496-1501 (2008).

79. Longair, M.H., Baker, D.A. \& Armstrong, J.D. Simple Neurite Tracer: open source software for reconstruction, visualization and analysis of neuronal processes. Bioinformatics 27, 2453-2454 (2011). 